\title{
Ramsey Properties of Countably Infinite Partial Orderings
}

\author{
Marcia J. Groszek * \\ Department of Mathematics \\ Dartmouth College \\ Hanover, NH, U.S.A. \\ marcia.groszek@dartmouth.edu
}

Submitted: May 5, 2011; Accepted: Feb 24, 2013; Published: Mar 1, 2013

Mathematics Subject Classifications: 05C55, 06A07

\begin{abstract}
A partial ordering $\mathbb{P}$ is chain-Ramsey if, for every natural number $n$ and every coloring of the $n$-element chains from $\mathbb{P}$ in finitely many colors, there is a monochromatic subordering $\mathbb{Q}$ isomorphic to $\mathbb{P}$. Chain-Ramsey partial orderings stratify naturally into levels. We show that a countably infinite partial ordering with finite levels is chain-Ramsey if and only if it is biembeddable with one of a canonical collection of examples constructed from certain edge-Ramsey families of finite bipartite graphs. A similar analysis applies to a large class of countably infinite partial orderings with infinite levels.
\end{abstract}

Keywords: Ramsey theory; partially ordered set; chain-Ramsey; edge-Ramsey

\section{Introduction}

A central definition of structural Ramsey theory is this:

Let $P, Q$, and $A$ be structures of the same type, and $k$ a natural number. Then

$$
P \rightarrow(Q)_{k}^{A}
$$

if, for every coloring in $k$ colors of the substructures of $P$ isomorphic to $A$, there is a monochromatic substructure of $P$ isomorphic to $Q$.

${ }^{*}$ The author thanks Jared Corduan, Joseph Mileti, and Peter Winkler for many fruitful conversations. 
(See, for example, the survey paper by Nešetrril [9].) One of the concerns of infinitary structural Ramsey theory is finding or classifying infinite structures $P$ such that, for a given $A$ and all natural numbers $k$, we have $P \rightarrow(P)_{k}^{A}$. We will denote this property by $P \rightarrow(P)^{A}$.

The original infinitary Ramsey's theorem [11] can be interpreted as $\mathbb{N} \rightarrow(\mathbb{N})^{n}$ for all $n$, where the natural numbers $\mathbb{N}$ are endowed with the usual ordering and $n$ denotes an $n$-element linear ordering, or as $K_{\omega} \rightarrow\left(K_{\omega}\right)^{K_{n}}$ for all $n$, where $K_{\omega}$ is the complete countably infinite graph and $K_{n}$ is the complete $n$-element graph.

The Rado graph, or countably infinite random graph, $G$ satisfies $G \rightarrow(G)^{A}$ when $A$ consists of a single vertex, but not when $A$ consists of two vertices connected by a single edge [4]. That is, $G$ is vertex-Ramsey but not edge-Ramsey. Both vertex-Ramseyness and edge-Ramseyness have been much investigated.

Milliken [6] shows that $\mathbb{P} \rightarrow(\mathbb{P})^{A}$ where $\mathbb{P}$ is the infinite binary tree equipped with the relations " $\sigma$ and $\tau$ are on the same level" and " $\gamma=\inf (\sigma, \tau)$," and $A$ is the complete binary tree of height $n$ with the same relations.

A consequence of this theorem, explicitly stated by Chubb, Hirst, and McNicholl [1], is that for all natural numbers $n, \mathbb{P} \rightarrow(\mathbb{P})^{n}$, where $\mathbb{P}$ is the infinite binary tree viewed as a partial ordering, and $n$ denotes an $n$-element linear ordering. We will call a partial ordering with this property chain-Ramsey. Chubb, Hirst, and McNicholl ask about other chain-Ramsey partial orderings.

This is a natural question. It is, perhaps, initially more appealing to ask which countably infinite partial orderings $\mathbb{P}$ satisfy $\mathbb{P} \rightarrow(\mathbb{P})^{A}$ for all finite partial orderings $A$. The answer is, not very many. In fact, up to biembeddability, there are only five. This can be proven from the related analysis of collections of finite partial orderings by Nešetřil [10], and we give a direct proof in Section 4. The difficulty Nešetřil identifies is that it is hard to satisfy $\mathbb{P} \rightarrow(\mathbb{P})^{A}$ if the structure $A$ is not rigid, or, indeed, has substructures that are not rigid. A partial ordering $A$ has no non-rigid substructures exactly in case $A$ is linear.

Thus it is natural to ask about colorings of chains. For instance, by work of Nešetřil and Rödl [7],[8], finite linear orderings are among a small class of structures $A$ for which, given any finite partial ordering $P$ and natural number $k$, there is a finite partial ordering $Q$ such that $Q \rightarrow(P)_{k}^{A}$. Fouché [5] investigates the finite combinatorics of this proposition.

Corduan, Groszek, and Mileti [3] show that if the partial ordering $\mathbb{P}$ is a countably infinite rooted tree, then $\mathbb{P}$ is chain-Ramsey if and only if $\mathbb{P}$ is biembeddable with either the natural numbers or the infinite binary tree, both with the usual orderings.

This paper considers the general question of which countably infinite partial orderings are chain-Ramsey.

In Section 2, we introduce some basic definitions, terminology and notation, much of which is standard, but not all. In particular, we define a bipartite graph $G$ with distinguished parts $M(G)$ and $S(G)$ to be extensional if no two elements of $S(G)$ have edges to exactly the same elements of $M(G)$. Equivalently, up to isomorphism, $S(G)$ is a collection of subsets of $M(G)$ and the edge relation is membership. The name extensional comes from the set-theoretic Axiom of Extensionality, which states that sets with the same members are equal. Extensional bipartite graphs will arise naturally in the investigation 
of chain-Ramsey partial orderings.

In Section 3, we show we can restrict our attention to a special class of partial orderings. First, if $\mathbb{P}$ is any countable partial ordering with the Ramsey property for 2-element chains, then either $\mathbb{P}$ or the reverse ordering $\mathbb{P}^{*}$ is $\omega$-linearizable. (To see this, let $\mathbb{P}=$ $\left\{p_{n} \mid n \in \mathbb{N}\right\}$, color 2-element chains $p_{i}<\mathbb{P} p_{j}$ red if $i<j$ and blue if $i>j$, and consider a monochromatic embedded copy of $\mathbb{P}$.) Since $\mathbb{P}$ and $\mathbb{P}^{*}$ share the same chainRamsey properties, we consider only $\omega$-linearizable partial orderings. Second, we define $\mathbb{P}$ and $\mathbb{Q}$ to be biembeddable if each is embeddable in the other. Biembeddability is an equivalence relation that preserves Ramsey properties. Hence, rather than individual partial orderings, we consider equivalence classes.

Since our partial orderings are $\omega$-linearizable, they are well-founded of height at most $\omega$, so they stratify into levels; level $n$ consists of all points of height $n$ in the partial ordering. We show that if $\mathbb{P}$ is a chain-Ramsey partial ordering with finite levels (meaning that there are finitely many points on each level), then up to biembeddability $\mathbb{P}$ has a least element, embeds into itself above any point, and contains no copy of the pentagon lattice $N 5$. We call partial orderings with these three properties proto-Ramsey.

In Section 4 we determine which proto-Ramsey partial orderings are chain-Ramsey. This answers the question of which countable partial orderings with finite levels are chainRamsey. Our analysis applies even if the levels of $\mathbb{P}$ are not finite, and answers the question of which countably infinite partial orderings with least element containing no copy of $N 5$ are chain-Ramsey. We do not know whether there are any chain-Ramsey partial orderings with least element that contain a copy of $N 5$. (If we do not require $\mathbb{P}$ to be connected, then there are such examples; one is the disjoint union of all finite partial orderings.)

To give a little more detail, we show that a proto-Ramsey partial ordering $\mathbb{P}$ naturally gives rise to a collection of extensional, connected bipartite graphs $\mathcal{G}(\mathbb{P})$. If $\mathbb{P}$ has finite levels, then all elements of $\mathcal{G}(\mathbb{P})$ are finite. The characterization theorem states that a proto-Ramsey partial ordering $\mathbb{P}$ is chain-Ramsey if and only if $\mathcal{G}(\mathbb{P})$ is edge-Ramsey and has the joint embedding property.

In Section 5 we show how to construct from a collection $\mathcal{G}$ of finite, extensional, connected bipartite graphs a proto-Ramsey partial ordering $\mathbb{P}(\mathcal{G})$ such that $\mathcal{G}(\mathbb{P}(\mathcal{G})$ ) is biembeddable with $\mathcal{G}$. (That is, each element of either collection is embeddable in some element of the other.) We say that $\mathcal{G}$ is singular if every element $G$ of $\mathcal{G}$ has only one vertex in $S(G)$, and $\mathbb{P}$ is singular if $\mathcal{G}(\mathbb{P})$ is singular. In the nonsingular case, there is a oneto-one correspondence between equivalence (biembeddability) classes of proto-Ramsey partial orderings with finite levels and equivalence (biembeddability) classes of collections of extensional, connected, finite bipartite graphs. Specifically, (up to biembeddability) $\mathcal{G}(\mathbb{P}(\mathcal{G}))=\mathcal{G}$, and (up to biembeddability) $\mathbb{P}(\mathcal{G}(\mathbb{P}))=\mathbb{P}$. In the singular case, each collection $\mathcal{G}$ corresponds to two equivalence classes of proto-Ramsey partial orderings, one with branching and one without; for example, the collection containing only the single graph consisting of two points connected by an edge corresponds to both $\omega$ (the natural numbers with the usual ordering) and the complete binary tree.

In Section 6, we exploit the correspondence between chain-Ramsey partial orderings and edge-Ramsey collections of bipartite graphs to generate an infinite sequence of col- 
lections of finite, connected, extensional bipartite graphs, such that each collection is edge-Ramsey and has the joint embedding property. This sequence corresponds to an infinite sequence of chain-Ramsey partial orderings.

In Section 7, we conclude with some questions.

\section{Notation and Terminology}

\subsection{Structures and Ramsey properties}

Definition 2.1. A structure $\mathbb{P}$ consists of a set $P$ equipped with some relations and functions. A substructure consists of a subset of $P$ with the restricted relations and functions. That is, substructure always means induced substructure.

Because we are interested in finite and countably infinite structures, unless otherwise specified, we will assume $P$ is a set of natural numbers.

We will often abuse notation by conflating $\mathbb{P}$ and $P$.

Definition 2.2. We say $P$ embeds into $Q$, written $P \hookrightarrow Q$, if there is an isomorphism from $P$ to a substructure of $Q$, where isomorphism preserves functions, relations and nonrelations. If both $P \hookrightarrow Q$ and $Q \hookrightarrow P$, then we say that $P$ and $Q$ are biembeddable and write $P \rightleftarrows Q$.

A relevant example is the following: The complete binary tree $2^{<\omega}$ and the complete infinitely branching tree $\omega^{<\omega}$, viewed as partial orderings, are biembeddable. Any embedding of $\omega^{<\omega}$ into $2^{<\omega}$ is an embedding of partial orderings only; it does not preserve infima.

For any substructure $Q$ of $P$, we have $P \rightleftarrows Q$ if and only if $P \hookrightarrow Q$.

Definition 2.3. If $\mathcal{C}$ and $\mathcal{C}^{\prime}$ are collections of structures, we say $\mathcal{C} \hookrightarrow \mathcal{C}^{\prime}$ if for every $Q \in \mathcal{C}$ there is $P \in \mathcal{C}^{\prime}$ such that $Q \hookrightarrow P$. If $\mathcal{C} \hookrightarrow \mathcal{C}^{\prime}$ and $\mathcal{C}^{\prime} \hookrightarrow \mathcal{C}$, we say $\mathcal{C}$ and $\mathcal{C}^{\prime}$ are biembeddable, $\mathcal{C} \rightleftarrows \mathcal{C}^{\prime}$.

Biembeddability, both of structures and of collections of structures, is an equivalence relation.

Definition 2.4. A collection $\mathcal{C}$ of structures has the joint embeddability property if for every $Q_{1}$ and $Q_{2}$ in $\mathcal{C}$, there is $P \in \mathcal{C}$ such that $Q_{1} \hookrightarrow P$ and $Q_{2} \hookrightarrow P$. We say $\mathcal{C}$ is j.e.p.

Definition 2.5. Let $P, Q$, and $A$ be structures of the same type, and $k$ a natural number.

$P^{[[A]]}$ is the set of all substructures of $P$ isomorphic to $A$.

An $A$-coloring of $P$ in $k$ colors is a function $c: P[[A]] \rightarrow\{0,1, \ldots, k-1\}$.

A substructure $P^{\prime}$ of $P$ is homogeneous for $c$, or monochromatic, if there is some $i<k$ such that $c\left[\left(P^{\prime}\right)^{[[A]]}\right]=\{i\}$. We say $P^{\prime}$ is homogeneous in color $i$. 
Definition 2.6. We say $P \rightarrow(Q)_{k}^{A}$ if for every $A$-coloring of $P$ in $k$ colors there is a monochromatic substructure of $P$ isomorphic to $Q$.

We say $P \rightarrow(Q)^{A}$ if $P \rightarrow(Q)_{k}^{A}$ for every natural number $k$.

If $P \rightarrow(P)^{A}$ we say $P$ is $A$-Ramsey.

Definition 2.7. A collection $\mathcal{C}$ of structures is $A$-Ramsey if for every $Q \in \mathcal{C}$ and every natural number $k$ there is $P \in \mathcal{C}$ such that $P \rightarrow(Q)_{k}^{A}$.

The property of being $A$-Ramsey is preserved by biembeddability, both of structures and of collections of structures.

In this paper our interest is in two cases. Either our structures are partial orderings and $A$ is a finite linear ordering, or our structures are bipartite graphs and $A$ consists of (two points joined by) a single edge.

\subsection{Partial orderings}

Definition 2.8. The natural number $n$, as a partial ordering, denotes an $n$-element linear ordering, also called a chain.

If $\mathbb{P}$ is a partial ordering and $\mathbb{P} \rightarrow(\mathbb{P})^{n}$, we say $\mathbb{P}$ has the $n$-Ramsey property, or $\mathbb{P}$ is $n$-Ramsey, and write $R^{n}(\mathbb{P})$. $R(\mathbb{P})$.

If $\mathbb{P}$ is $n$-Ramsey for every natural number $n$, we say $\mathbb{P}$ is chain-Ramsey, and write

Because biembeddability preserves Ramsey properties, we can characterize the chainRamsey partial orderings by characterizing their biembeddability classes.

Definition 2.9. If $\mathbb{P}=\langle P, \leqslant\rangle$ is a partial ordering, $\mathbb{P}^{*}=\langle P, \geqslant\rangle$.

Definition 2.10. The partial ordering $\omega$ consists of the natural numbers $\{0,1,2, \ldots\}$ with the usual ordering.

Definition 2.11. A countably infinite partial ordering $\mathbb{P}=\left\langle P, \leqslant_{\mathbb{P}}\right\rangle$ is $\omega$-linearizable [or $\omega^{*}$-linearizable] if the elements of $P$ can be enumerated as $\left\{p_{n} \mid n \in \omega\right\}$ such that if $p_{m}<\mathbb{P} p_{n}$ then $m<n$ [or $\left.m>n\right]$.

If $\mathbb{P}=\left\langle P, \leqslant_{\mathbb{P}}\right\rangle$ is $\omega$-linearizable, we may assume that $P \subseteq \omega$, and that if $p<_{\mathbb{P}} q$ then $p<q$.

Definition 2.12. An $\omega$-linearizable partial ordering $\mathbb{P}$ is stratified into levels, defined recursively by:

Level 0 contains all minimal points.

Level $n+1$ contains all points not on earlier levels all of whose predecessors (in the $\leqslant_{\mathbb{P}}$ ordering) are on earlier levels.

Level $n$ of $\mathbb{P}$ is denoted $\mathbb{P}_{n}$, and if $p \in \mathbb{P}_{n}$ then $\operatorname{lev}(p)=n$.

$\mathbb{P}_{\leqslant n}=\bigcup_{m \leqslant n} \mathbb{P}_{m}$ and $\mathbb{P}_{<n}=\bigcup_{m<n} \mathbb{P}_{m}$

$\operatorname{Pred}(p)=\left\{q \in \mathbb{P} \mid q<_{\mathbb{P}} p\right\}$.

If $n<\operatorname{lev}(p)$ then $\operatorname{Pred}_{n}(p)=\left\{q \in \mathbb{P}_{n} \mid q<_{\mathbb{P}} p\right\}$. 
Definition 2.13. An $\omega$-linearizable partial ordering $\mathbb{P}$ is a finite-level partial ordering if all its levels are finite.

We will give a complete characterization of the biembeddability classes of countably infinite, finite-level chain-Ramsey partial orderings.

\subsection{Bipartite graphs}

Definition 2.14. A bipartite graph $G=\langle M(G), S(G), E(G)\rangle$ consists of disjoint sets of vertices $M(G)$ and $S(G)$, and a set of edges $E(G) \subseteq M(G) \times S(G)$.

Our bipartite graphs have distinguished parts, so an embedding of $G$ into $H$ must send $M(G)$ to $M(H)$ and $S(G)$ to $S(H)$.

Definition 2.15. As a bipartite graph, $e$ denotes the graph consisting of two vertices connected by a single edge. For $e$-Ramsey we may write edge-Ramsey.

Definition 2.16. A bipartite graph $G$ is extensional if no two distinct points in $S(G)$ have edges to exactly the same points in $M(G)$. Equivalently, up to isomorphism, the points in $S(G)$ are subsets of $M(G)$ and the edge relation is membership.

The notation $S(G)$ and $M(G)$ is intended to suggest sets and members. The term extensional comes from the set-theoretic Axiom of Extensionality, which states that sets with exactly the same members must be equal.

Definition 2.17. For $p$ and $q$ in $S(G)$, define $p \equiv_{M} q$ if and only if $p$ and $q$ are connected to exactly the same points in $M(G)$.

A maximal extensional subgraph of $G$ is a subgraph $H$ such that $M(H)=M(G)$ and $S(H)$ contains exactly one element from each $\equiv_{M}$-equivalence class of $S(G)$.

As all maximal extensional subgraphs of $G$ are naturally isomorphic, we may speak of the maximal extensional subgraph of $G$.

We will be concerned with connected, extensional bipartite graphs because they arise naturally in the analysis of chain-Ramsey partial orderings. For the same reason we will be concerned with collections that are closed under (connected extensional) subgraphs and have the joint embedding property. However, the following proposition indicates that even if our interest were in edge-Ramsey collections of bipartite graphs, it would make some sense to consider j.e.p. collections of connected, extensional bipartite graphs.

Proposition 2.18. Let $\mathcal{G}$ be a collection of bipartite graphs.

The collection $\mathcal{G}$ is edge-Ramsey if and only if $\mathcal{G}$ is a union of j.e.p. edge-Ramsey collections.

If $\mathcal{G}$ is edge-Ramsey, so are the following:

1. The collection of all subgraphs of elements of $\mathcal{G}$.

2. The collection of all connected subgraphs of elements of $\mathcal{G}$. 
3. The collection of all connected, extensional subgraphs of elements of $\mathcal{G}$.

4. The collection of all connected graphs whose maximal extensional subgraph can be embedded in an element of $\mathcal{G}$.

5. If every element of $\mathcal{G}$ is finite, the collection of all finite connected graphs whose maximal extensional subgraph can be embedded in an element of $\mathcal{G}$.

This is mostly well-known and standard. For questions involving extensional subgraphs, note that a coloring $c$ of $G$ naturally induces a coloring of the maximal extensional subgraph of $G$, by coloring an edge $(p, s)$ a color that appears at least as often as any other among the $c(p, t)$ with $t \equiv_{M} s$.

Definition 2.19. If $\mathcal{G}$ is a collection of bipartite graphs, $\mathcal{C} \ell(\mathcal{G})$ is the collection of all connected, extensional bipartite graphs $H$ with $M(H) \subseteq \omega$ and $S(H) \subseteq \omega$ that can be embedded in elements of $\mathcal{G}$.

Claim 2.20. If $\mathcal{G}$ is a collection of bipartite graphs, $\mathcal{C} \ell(\mathcal{C} \ell(\mathcal{G}))=\mathcal{C} \ell(\mathcal{G})$. If all elements of $\mathcal{G}$ are connected and extensional, then $\mathcal{G} \rightleftarrows \mathcal{C} \ell(\mathcal{G})$. Furthermore, if also all elements of $\mathcal{H}$ are connected and extensional, then $\mathcal{G} \hookrightarrow \mathcal{H}$ if and only if $\mathcal{C} \ell(\mathcal{G}) \subseteq \mathcal{C} \ell(\mathcal{H})$, and $\mathcal{G} \rightleftarrows \mathcal{H}$ if and only if $\mathcal{C} \ell(\mathcal{G})=\mathcal{C} \ell(\mathcal{H})$.

\section{Countable Chain-Ramsey Partial Orderings}

In this section, we develop some structural properties of countably infinite chain-Ramsey partial orderings.

From the known proof that the rationals do not have the 2,2-Ramsey property, we can derive the following fact [3].

Proposition 3.1. If $\mathbb{P}$ is a countably infinite partial ordering and $R^{2}(\mathbb{P})$ holds, then $\mathbb{P}$ is either $\omega$-linearizable or $\omega^{*}$-linearizable.

Proof. Assume $\mathbb{P} \subseteq \omega$. Color 2-element chains from $\mathbb{P}$ according to whether or not the $<_{\mathbb{P}}$ ordering agrees with the usual ordering $<$ on $\omega$; for $p<\mathbb{P} q$,

$$
c(p, q)= \begin{cases}0 & p<q \\ 1 & p>q\end{cases}
$$

Let $H$ be a homogeneous subordering isomorphic to $\mathbb{P}$. Suppose that $H$ is homogeneous in color 0 , that is, for $\langle p, q\rangle \in H^{[2]]}$ we have $c(p, q)=0$. Then the enumeration of $H$ in its natural ordering shows $H$ is $\omega$-linearizable. If $H$ is homogeneous in color 1 , then $H$ is $\omega^{*}$-linearizable. Since $H$ is isomorphic to $\mathbb{P}$, this shows that $\mathbb{P}$ is either $\omega$-linearizable or $\omega^{*}$-linearizable.

If $\mathbb{P}$ is $\omega^{*}$-linearizable, then $\mathbb{P}^{*}$ is $\omega$-linearizable. As $\mathbb{P}^{*}$ is chain-Ramsey if and only if $\mathbb{P}$ is, we will restrict our attention to $\omega$-linearizable partial orderings. 
Definition 3.2. Points $p$ and $q$ in $\mathbb{P}$ are compatible if they have a common upper bound $r\left(p \leqslant_{\mathbb{P}} r\right.$ and $\left.q \leqslant_{\mathbb{P}} r\right)$, and incompatible otherwise. If $p$ and $q$ are incompatible we write $p \perp q$.

Lemma 3.3. Suppose that $\mathbb{P}$ is an $\omega$-linearizable finite-level partial ordering and $R^{3}(\mathbb{P})$ holds. Then for any $p$ in $\mathbb{P}$, there is an $n \in \omega$ such that if $p_{0}<_{\mathbb{P}} p_{1}<_{\mathbb{P}} \cdots<_{\mathbb{P}} p_{n}$ is any increasing chain, either $p \leqslant_{\mathbb{P}} p_{n}$ or $p \perp p_{n}$.

Proof. Assume $\mathbb{P} \subseteq \omega$. Color 3-chains $a<\mathbb{P} b<_{\mathbb{P}} c$ as follows: For $q<\mathbb{P} r$, let $i(q, r)$ be the least $i$ (in the sense of the usual ordering $<$ on $\omega$ ) such that $i \mathbb{\mathbb { P }}_{\mathbb{P}} q$ but $i \leqslant_{\mathbb{P}} r$. Color the chain $\langle a, b, c\rangle$ color 0 if $i(a, b)<i(b, c)$ and color 1 if $i(a, b)>i(b, c)$.

Let $\varphi$ be an isomorphism of $\mathbb{P}$ onto a monochromatic isomorphic subordering $\mathbb{Q}$. As the finite-level chain-Ramsey partial ordering $\mathbb{Q}$ must have an infinite increasing chain $q_{0}<\mathbb{P}$ $q_{1}<\mathbb{P} \ldots, \mathbb{Q}$ must be homogeneous in color 0 ; we must have $i\left(q_{k}, q_{k+1}\right)<i\left(q_{k+1}, q_{k+2}\right)$ rather than the reverse.

Now let $q=\varphi(p)$ and $n=q+2$. Suppose toward a contradiction that $p \mathbb{E}_{\mathbb{P}} p_{n}$ and $p \not \perp p_{n}$ for some increasing chain $p_{0}<_{\mathbb{P}} p_{1}<_{\mathbb{P}} \cdots<_{\mathbb{P}} p_{n}$. Let $q_{k}=\varphi\left(p_{k}\right)$ for $k \leqslant n$. Then $q \mathbb{P}_{\mathbb{P}} q_{n}$ and $q \not \perp q_{n}$ in $\mathbb{Q}$. Because the function $i$ increases along chains, $i\left(q_{n-1}, q_{n}\right) \geqslant n-1>q$. Since $q \not \perp q_{n}$, let $r$ be a common upper bound in $\mathbb{Q}$. Then as $q \mathbb{P}_{\mathbb{P}} q_{n}$ but $q \leqslant \mathbb{P} r$, we have $i\left(q_{n}, r\right) \leqslant q<i\left(q_{n-1}, q_{n}\right)$. This means the chain $\left\langle q_{n-1}, q_{n}, r\right\rangle$ has color 1 , a contradiction.

Proposition 3.4. Suppose that $\mathbb{P}$ is an $\omega$-linearizable finite-level partial ordering and $R^{2}(\mathbb{P})$ and $R^{3}(\mathbb{P})$ hold. Then the following forbidden configuration does not occur in $\mathbb{P}$ : points $p$ and $q$ that are incomparable but compatible with common successor $s$, and a point $r$ that is below $p$ but not below $q$.

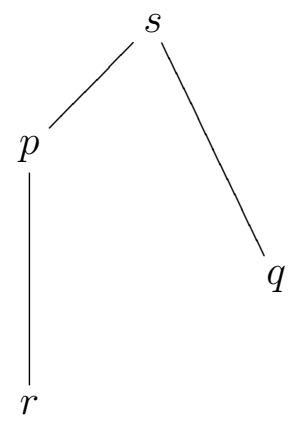

Proof. By Lemma 3.3, for every $p$ in $\mathbb{P}$ there is a level $h(p)$ such that any point on level $h(p)$ or above must be either above or incompatible with $p$. Because the levels of $\mathbb{P}$ are finite, we can define a function $f$ as follows: $f(0)=0$; given $f(n)$, choose $f(n+1)$ so that for any point $p$ on or below level $f(n)$, we have $h(p)<f(n+1)$.

Color chains $p<_{\mathbb{P}} q$ as follows: Let $n$ be least such that $p$ is below level $f(n)$. Then the chain $\langle p, q\rangle$ has color 0 if $q$ is above level $f(n+2)$, and color 1 otherwise.

Let $\mathbb{Q}$ be an isomorphic monochromatic subordering. Because $\mathbb{Q}$ has infinite height, it must be monochromatic in color 0 . Suppose toward a contradiction that $p, q, s, r$ realize 
the forbidden configuration in $\mathbb{Q}$, and let $n$ be least such that the level of $r$ is below $f(n)$. By definition of $f$, as $r$ and $q$ are incomparable but compatible, the level of $q$ is below $f(n+1)$, and as $p$ and $q$ are incomparable but compatible, the level of $p$ is below level $f(n+2)$. But this means the chain $\langle r, p\rangle$ has color 1 , a contradiction.

This forbidden configuration, when augmented by a bottom point, becomes a copy of the pentagon lattice $N 5$. As we will very soon restrict our attention to partial orderings with a least element, we make the following definition.

Definition 3.5. A partial ordering with least element in which this forbidden configuration does not occur is called $N 5$-omitting.

Proposition 3.6. Suppose that $\mathbb{P}$ is a partial ordering with finitely many minimal elements, and $R^{1}(\mathbb{P})$ holds. Then $\mathbb{P}$ is biembeddable with a partial ordering $\mathbb{Q}$ with a least element. Furthermore, if $\mathbb{P}$ is finite-level or omits the forbidden configuration, the same holds for $\mathbb{Q}$.

Proof. By assumption, $\mathbb{P}$ has finitely many minimal nodes. Color the nodes of $\mathbb{P}$ according to the least (in the sense of the ordering on $\omega$ ) minimal node below them. By $R^{1}(\mathbb{P})$ there are a minimal node $p_{0}$ and an embedding $\varphi$ of $\mathbb{P}$ into $\left\{p \mid p \geqslant \mathbb{P} p_{0}\right\}$. Let $\mathbb{Q}=$ $\operatorname{range}(\varphi) \cup\left\{p_{0}\right\}$. Then $\mathbb{P} \rightleftarrows \mathbb{Q}$.

Definition 3.7. A point $p$ of a partial ordering $\mathbb{P}$ is a base of $\mathbb{P}$ if there is an embedding $\varphi$ of $\mathbb{P}$ into $\{q \mid p \leqslant \mathbb{P} q\}$. The embedding $\varphi$ witnesses that $p$ is a base of $\mathbb{P}$.

The partial ordering $\mathbb{P}$ is densely self-embeddable if every point of $\mathbb{P}$ is a base of $\mathbb{P}$.

Note that the bases of $\mathbb{P}$ form a downward-closed suborder.

Proposition 3.8. Suppose that $\mathbb{P}$ is a partial ordering with least element $p_{0}$ and $R^{1}(\mathbb{P})$ holds. Then $\mathbb{P}$ is biembeddable with a densely self-embeddable $\mathbb{Q}$ with least element. Furthermore, if $\mathbb{P}$ is finite-level or omits the forbidden configuration, the same holds for $\mathbb{Q}$.

Proof. Let $\mathbb{Q}$ consist of all the bases of $\mathbb{P}$. Then $p_{0}$ is also the least element of $\mathbb{Q}$, and $\mathbb{Q}$ is downward closed in $\mathbb{P}$, hence if $\mathbb{P}$ is finite-level so is $\mathbb{Q}$. Color $\mathbb{P}$ by giving color 0 to the elements of $\mathbb{Q}$, and color 1 to all other points. Let $\varphi$ be an embedding of $\mathbb{P}$ with monochromatic range. Clearly $\varphi\left(p_{0}\right)$ is a base of $\mathbb{P}$, so $\varphi$ embeds $\mathbb{P}$ into $\mathbb{Q}$, showing $\mathbb{P} \rightleftarrows \mathbb{Q}$.

To see that $\mathbb{Q}$ is densely self-embeddable, first note that any embedding $\varphi: \mathbb{P} \rightarrow \mathbb{P}$ must send $\mathbb{Q}$ to $\mathbb{Q}$ : Suppose that $\psi: \mathbb{P} \rightarrow \mathbb{P}$ witnesses that $q$ is a base of $\mathbb{P}$. Then $\varphi \circ \psi$ witnesses that $\varphi(q)$ is a base of $\mathbb{P}$; that is, $\varphi(q) \in \mathbb{Q}$.

But now, if $\varphi$ witnesses that $r$ is a base of $\mathbb{P}$, then $\varphi \uparrow \mathbb{Q}$ witnesses that $r$ is a base of $\mathbb{Q}$. Hence every element of $\mathbb{Q}$ is a base of $\mathbb{Q}$, so $\mathbb{Q}$ is densely self-embeddable.

Definition 3.9. A partial ordering $\mathbb{P}$ is proto-Ramsey if $\mathbb{P}$ is $\omega$-linearizable, $N 5$-omitting, and densely self-embeddable, and has a least element.

A partial ordering $\mathbb{P}$ is weakly proto-Ramsey if $\mathbb{P}$ is $\omega$-linearizable and N5-omitting, and has a least element (but is not necessarily densely self-embeddable). 
By Proposition 3.8, if $\mathbb{P}$ is weakly proto-Ramsey and chain-Ramsey, then $\mathbb{P}$ is biembeddable with a proto-Ramsey, chain-Ramsey partial ordering.

Proposition 3.10. If a finite-level partial ordering is chain-Ramsey, then it is biembeddable with a proto-Ramsey finite-level partial ordering.

Proof. This follows from Propositions 3.4, 3.6, and 3.8, using only $R^{n}(\mathbb{P})$ for $n \leqslant 3$.

Our task now becomes to determine which proto-Ramsey partial orderings are chainRamsey. We will do this in Section 4. Noting some structural properties of proto-Ramsey partial orderings will be useful.

Proposition 3.11. Suppose that $\mathbb{P}$ is a weakly proto-Ramsey partial ordering. Then any two incomparable but compatible points of $\mathbb{P}$ must have exactly the same predecessors. It follows that:

If $p$ and $q$ are incomparable but compatible, then $p$ and $q$ are on the same level, and have a common successor on the next level.

Predecessors of $p$ on different levels must be comparable. The predecessors of $p$ are in a sense almost linearly ordered: If $q<_{\mathbb{P}} p$ has level $n$, and $r<_{\mathbb{P}} p$ has level $m<n$, then $r<_{\mathbb{P}} q$.

If $p$ is above level $n$, and $p \leqslant_{\mathbb{P}} q$, then $p$ and $q$ have the same predecessors on level $n$.

If $p$ is above level $n$, then $\operatorname{Pred}_{n}(p)=\operatorname{Pred}_{n}(q)$ for some $q \in \mathbb{P}_{n+1}$.

The ordering $<_{\mathbb{P}}$ is the transitive closure of $\left\{\langle p, q\rangle \mid p<_{\mathbb{P}} q \& \operatorname{lev}(q)=\operatorname{lev}(p)+1\right\}$.

In general, the structure and complexity of a weakly proto-Ramsey partial ordering are completely determined by the ordering between successive levels.

\section{Edge-Ramsey Collections of Finite Bipartite Graphs}

Throughout this section, $\mathbb{P}$ will denote a weakly proto-Ramsey partial ordering. From $\mathbb{P}$ we define a collection $\mathcal{G}(\mathbb{P})$ of bipartite graphs, such that $\mathcal{C} \ell(\mathcal{G})$ depends only on the biembeddability class of $\mathbb{P}$. We will show that if $\mathbb{P}$ is proto-Ramsey, then $\mathbb{P}$ is chainRamsey if and only if $\mathcal{G}(\mathbb{P})$ is edge-Ramsey and has the joint embedding property.

\section{1 $\mathcal{G}(\mathbb{P})$ and colorings of $\mathbb{P}$}

Definition 4.1. Let $p$ and $q$ be in $\mathbb{P}_{n}$. Define $p \equiv_{p r} q$ if $\operatorname{Pred}(p)=\operatorname{Pred}(q)$, with the equivalence class of $p$ denoted $[p]_{p r}$. Define $\equiv$ to be the transitive closure of the compatibility relation on $\mathbb{P}_{n}$ (where $p$ and $q$ are compatible if they have a common successor), with the equivalence class of $p$ denoted $[p]$.

Because $\mathbb{P}$ is $N 5$-omitting, each $\equiv$ class is contained within a single $\equiv_{p r}$ class. By definition, each set $s=\operatorname{Pred}_{n}(p)$ is contained within a single $\equiv$ class. 
Definition 4.2. For each $\equiv$ class $a=[p]$ for $p \in \mathbb{P}_{n}$, define a connected extensional bipartite graph $G_{a}$. Set $M\left(G_{a}\right)=a$ and $S\left(G_{a}\right)=\left\{s \subseteq a \mid(\exists q)\left(\operatorname{Pred}_{n}(q)=s\right)\right\}$. The edge relation of $G_{a}$ is membership.

Because $\mathbb{P}$ is $\omega$-linearizable, even if $\mathbb{P}$ is not finite-level, each set $\operatorname{Pred}_{n}(q) \operatorname{must}_{\text {be }}$ finite.

Definition 4.3. $\mathcal{G}(\mathbb{P})=\left\{G_{[p]} \mid p \in \mathbb{P}\right\}$.

In the rest of this section, we show that if $\mathbb{P}$ is proto-Ramsey, then $\mathbb{P}$ is chain-Ramsey if and only if $\mathcal{G}(\mathbb{P})$ is edge-Ramsey and has the joint embedding property.

Definition 4.4. If $\mathcal{G}$ is a collection of bipartite graphs, an edge coloring of $\mathcal{G}$ in $k$ colors is a collection $\left\{c_{G}: E(G) \rightarrow\{0,1, \ldots, k-1\} \mid G \in \mathcal{G}\right\}$.

In the above definition, $\mathcal{G}$ must be understood as a collection of individual graphs rather than isomorphism types. Isomorphic elements of $\mathcal{G}$ may be colored differently.

Definition 4.5. If $c$ is an edge coloring of $\mathcal{G}(\mathbb{P})$, and $n>1$, then $c$ induces a coloring $\bar{c}$ of $n$-element chains in $\mathbb{P}$ by

$$
\bar{c}\left(p_{1}, p_{2}, \ldots, p_{n}\right)=c_{G_{\left[p_{1}\right]}}\left(p_{1}, \operatorname{Pred}_{l e v e l\left(p_{1}\right)}\left(p_{2}\right)\right) .
$$

Such a coloring is called graph-induced.

If $c$ is an edge coloring of $\mathcal{G}(\mathbb{P})$ such that, for $\langle q, s\rangle \in G_{a}$, the color $c_{G_{a}}(q, s)$ depends only on $q$, and we denote that color by $c_{G_{a}}(q)$, then $c$ induces a coloring $\bar{c}$ of 1 -tuples in $\mathbb{P}$ by $\bar{c}(p)=c_{G_{[p]}}(p)$.

Remark 4.6. Any coloring $\bar{c}$ of $n$-element chains in $\mathbb{P}$ such that $\bar{c}\left(p_{1}, p_{2}, \ldots, p_{n}\right)$ depends only on $p_{1}$ and $\operatorname{Pred}_{l e v\left(p_{1}\right)}\left(p_{2}\right)$ is graph-induced, and $\bar{c}$ determines the corresponding edge coloring $c$ of $\mathcal{G}(\mathbb{P})$ by $c_{G_{a}}(p, s)=\bar{c}\left(p, q, r_{3}, \ldots r_{n}\right)$, where $\operatorname{Pred}_{l e v(p)}(q)=s$.

Proposition 4.7. An embedding $\varphi: \mathbb{P} \rightarrow \mathbb{P}$ induces an embedding of $G_{[p]}$ into $G_{[\varphi(p)]}$. If the range of $\varphi$ is homogeneous for some coloring $\bar{c}$ induced by an edge coloring $c$ of $\mathcal{G}(\mathbb{P})$, then the image of $G_{[p]}$ is a subgraph of $G_{[\varphi(p)]}$ homogeneous for $c_{G_{[\varphi(p)]}}$. Conversely, if, for some $i$, each image of each $G_{[p]}$ is homogeneous for $c_{G_{[\varphi(p)]}}$ in color $i$, then the range of $\varphi$ is homogeneous for $\bar{c}$ in color $i$.

Proof. Define $k: G_{[p]} \rightarrow G_{[\varphi(p)]}$ as follows: For $q \in M\left(G_{[p]}\right)=[p]$ let $k(q)=\varphi(q)$. For $s \in S\left(G_{[p]}\right)$, choose $r$ such that $s=\operatorname{Pred}_{l e v(p)}(r)$, and let $k(s)=\operatorname{Pred}_{l e v(\varphi(p))}(\varphi(r))$.

(This embedding is not necessarily unique; if also $s=\operatorname{Pred}_{l e v(p)}\left(r^{\prime}\right)$, and $r \perp r^{\prime}$, we can have $\operatorname{Pred}_{l e v(\varphi(p))}(\varphi(r)) \neq \operatorname{Pred}_{l e v(\varphi(p))}\left(\varphi\left(r^{\prime}\right)\right)$, as long as their difference lies outside the range of $\varphi$.)

The edge in $G_{[\varphi(p)]}$ between $k(p)=\varphi(p)$ and $k(s)=\operatorname{Pred}_{l e v(\varphi(p))}(\varphi(r))$ is assigned color $c_{G_{[\varphi(p)]}}\left(\varphi(p), \operatorname{Pred}_{l e v(\varphi(p))}(\varphi(r))\right)=\bar{c}\left(\varphi(p), \varphi(r), p_{3}, \ldots, p_{n}\right)$ for any $p_{3}, \ldots, p_{n}$ in the range of $\varphi$ above $\varphi(r)$, so if the range of $\varphi$ is monochromatic in color $i$, so is the range of $k$. Similarly, if the range of $k$ is monochromatic in color $i$ for all $k$, then the range of $\varphi$ is monochromatic in color $i$. 
Proposition 4.8. Let $\mathbb{P}$ be a weakly proto-Ramsey partial ordering. If $\mathbb{P}$ is chain-Ramsey, then $\mathcal{G}(\mathbb{P})$ is edge-Ramsey and has the joint embedding property.

Proof. If $\mathcal{G}(\mathbb{P})$ fails to have the joint embedding property, choose $G_{[p]}$ and $G_{[q]}$ such that no element of $\mathcal{G}(\mathbb{P})$ contains copies of both $G_{[p]}$ and $G_{[q]}$. Edge color $\mathcal{G}(\mathbb{P})$ by coloring all edges of $G$ color 0 if $G_{[p]} \hookrightarrow G$, and color 1 otherwise, which induces a coloring $\bar{c}$ of singletons in $\mathbb{P}$. Any embedding $\varphi$ of $\mathbb{P}$ into itself must send $G_{[p]}$ to a graph with color 0 edges, and $G_{[q]}$ to a graph with color 1 edges, and so the range of $\varphi$ cannot be homogeneous. Hence $R^{1}(\mathbb{P})$ fails.

If $\mathcal{G}(\mathbb{P})$ fails to be edge-Ramsey, choose $G_{[p]}$ and $k$ such that for each $G \in \mathcal{G}(\mathbb{P})$ there is a coloring $c_{G}$ of the edges of $G$ in $k$ colors such that $G$ contains no homogeneous copy of $G_{[p]}$. The collection of $c_{G}$ is an edge coloring of $\mathcal{G}(\mathbb{P})$, which induces a coloring $\bar{c}$ of pairs in $\mathbb{P}$. Any embedding $\varphi$ of $\mathbb{P}$ into itself must send $G_{[p]}$ to a graph that is not homogeneous, and so the range of $\varphi$ cannot be homogeneous. Hence $R^{2}(\mathbb{P})$ fails.

In the rest of this section we prove the converse to this proposition for proto-Ramsey $\mathbb{P}$. As a corollary we will see that for finite-level partial orderings $\mathbb{P}$ we have $R^{3}(\mathbb{P}) \Longrightarrow R(\mathbb{P})$.

\subsection{Embeddings of weakly proto-Ramsey partial orderings}

Definition 4.9. Points $p$ and $q$ in an $\omega$-linearizable partial ordering $\mathbb{P}$ are strongly incompatible, written $p \perp_{s t} q$, if for any $p^{\prime} \equiv p$ and $q^{\prime} \equiv q$, we have $p^{\prime} \perp q^{\prime}$.

Remark 4.10. Equivalently, if $\operatorname{lev}(p) \leqslant \operatorname{lev}(q)$ and $r \leqslant \mathbb{P} q$ has the same level as $p$, then $p \perp_{s t} q$ if and only if $p \not \equiv r$, and symmetrically if $\operatorname{lev}(q) \leqslant \operatorname{lev}(p)$.

If $p \perp q, p<_{\mathbb{P}} p^{\prime}$ and $q<_{\mathbb{P}} q^{\prime}$, then $p^{\prime} \perp_{s t} q^{\prime}$.

Proposition 4.11. Let $\mathbb{P}$ and $\mathbb{Q}$ be weakly proto-Ramsey partial orderings. If

(1.) $(\forall q \in \mathbb{Q})(\forall p \in \mathbb{P})(\exists r \geqslant \mathbb{P} p)\left(G_{[q]} \hookrightarrow G_{[r]}\right)$, and

(2.) every element of $\mathbb{P}$ has incompatible successors,

then there is an embedding from $\mathbb{Q}$ into $\mathbb{P}$.

Before proving Proposition 4.11, we make some comments and give a relevant definition.

Notice that if $\mathbb{P}$ is in fact proto-Ramsey, then $\mathcal{G}(\mathbb{Q}) \hookrightarrow \mathcal{G}(\mathbb{P})$ implies (1). Also, if $\mathbb{P}$ is proto-Ramsey, then (1) implies (2), provided there is at least one $G_{u}$ in $\mathcal{G}(\mathbb{P})$ for which $S\left(G_{u}\right)$ contains more than one element, or provided that $\mathbb{P}$ has at least one pair of incompatible points.

The idea behind the proof of Proposition 4.11 is as follows. We wish to define $j$ embedding $\mathbb{Q}$ into $\mathbb{P}$ level-by-level, choosing $j(q)$ for $q \in \mathbb{Q}_{n}$ and then choosing $j(r)$ for $r \in \mathbb{Q}_{n+1}$. In order to do this, if, for example, $r>_{\mathbb{Q}} q_{1}, q_{2}, r \ngtr_{\mathbb{Q}} q_{3}$, then there must be some point $p>_{\mathbb{P}} j\left(q_{1}\right), j\left(q_{2}\right), p \ngtr_{\mathbb{P}} j\left(q_{3}\right)$ to choose as $j(r)$. In fact, by Proposition 4.7, the embedding $j\left\lceil[q]\right.$ must extend to an embedding $k: G_{[q]} \rightarrow G_{[j(q)]}$.

It turns out that the existence of suitable embeddings $k$ for each $G_{[q]}$ is precisely what we need to appropriately define $j$ on $\mathbb{Q}_{n+1}$. Hence we make the following definition. 
Definition 4.12. An extendible $n$-embedding of $\mathbb{Q}$ into $\mathbb{P}$ is an embedding $j: \mathbb{Q} \leqslant n \rightarrow \mathbb{P}$ such that:

(1.) For $m \leqslant n$ and $p, q \in \mathbb{Q}_{m}$,

(a.) if $p \not \equiv q$ then $j(p) \perp_{s t} j(q)$,

(b.) if $p \equiv q$ then $j(p) \equiv j(q)$.

By (b), if $a=[p]$, we can unambiguously set $j(a)=[j(p)]$.

(2.) There is, for each $m \leqslant n$ and $[q] \subseteq \mathbb{Q}_{m}$, an embedding $k_{[q]}$ of $G_{[q]}$ to $G_{[j(q)]}$ satisfying:

(a.) $k_{[q]} \uparrow[q]=j \uparrow[q]$ (recall that $[q]=M\left(G_{[q]}\right)$ ),

(b.) if $s=\operatorname{Pred}_{m}(r) \in S\left(G_{[q]}\right)$ for $q<r \in \mathbb{Q}_{\leqslant n}$, then $k_{[q]}(s)=\operatorname{Pred}_{l e v(j(q))}(j(r))$.

Proof of Proposition 4.11. We define an embedding from $\mathbb{Q}$ to $\mathbb{P}$ as the limit of a sequence of extendible $n$-embeddings.

If $q_{0}$ is the least element of $\mathbb{Q}$, then $G_{\left[q_{0}\right]}$ consists of two vertices connected by an edge. We can define an extendible 0 -embedding by choosing $j\left(q_{0}\right)$ arbitrarily, setting $k_{\left[q_{0}\right]}\left(q_{0}\right)=j\left(q_{0}\right)$, and, for the unique element $s$ of $S\left(G_{\left[q_{0}\right]}\right)$, choosing $k_{\left[q_{0}\right]}(s)$ to be any element of $S\left(G_{\left[j\left(q_{0}\right)\right]}\right)$ connected to $j\left(q_{0}\right)$ by an edge. (Equivalently, choose $r>_{\mathbb{P}} j\left(q_{0}\right)$, and set $k_{\left[q_{0}\right]}(s)=\operatorname{Pred}_{l e v\left(j\left(q_{0}\right)\right)}(r)$.)

We will show that every extendible $n$-embedding can be extended to an extendible $(n+1)$-embedding.

Suppose, then, that the functions $k_{[p]}$ for $[p] \subseteq \mathbb{Q} \leqslant n$ witness that $j$ is an extendible $n$-embedding. We wish to extend $j$ to $\bar{j}$ on $\mathbb{Q}_{n+1}$ and define functions $\bar{k}_{[q]}$ for $[q] \subseteq \mathbb{Q}_{n+1}$ witnessing that $\bar{j}$ is extendible.

We define $\bar{j}$ and the $\bar{k}_{[q]}$ as follows.

1. Each set $\operatorname{Pred}_{n}(q)$ for $q \in \mathbb{Q}_{n+1}$ appears as an element $s \in S\left(G_{[p]}\right)$ for a unique $[p] \subseteq \mathbb{Q}_{n}$. Suppose that $k_{[p]}(s)=\operatorname{Pred}_{l e v(j(p))}(r)$. Then by condition $(2)$ in the definition of $n$-extendible, for $p^{\prime} \in[p], j\left(p^{\prime}\right)=k_{[p]}\left(p^{\prime}\right)<_{\mathbb{P}} r$ if and only if $p^{\prime}<_{\mathbb{Q}} q$, and by condition (1), for $p^{\prime} \in \mathbb{Q}_{n}-[p], j\left(p^{\prime}\right) \nless_{\mathbb{P}} r$. For each $s$ choose such a $r$, such that $k_{[p]}(s)=\operatorname{Pred}_{l e v(j(p))}(r)$, denoted $r_{s}$.

If $s \neq s^{\prime}$ then $r_{s}$ and $r_{s^{\prime}}$ are incompatible: Choose $p \in s$ and $p^{\prime} \in s^{\prime}$, so $s \in S\left(G_{[p]}\right)$ and $s^{\prime} \in S\left(G_{\left[p^{\prime}\right]}\right)$. We have that $j(p)<_{\mathbb{P}} r_{s}$ and $j\left(p^{\prime}\right)<_{\mathbb{P}} r_{s^{\prime}}$. If $p \not \equiv p^{\prime}$ then by condition (1) $r_{s} \perp r_{s^{\prime}}$. If $p \equiv p^{\prime}$, then because $j(p) \equiv j\left(p^{\prime}\right)$, we have $\operatorname{lev}(j(p))=\operatorname{lev}\left(j\left(p^{\prime}\right)\right)$. Because $s$ and $s^{\prime}$ contain different points, $r_{s}$ and $r_{s^{\prime}}$ have different predecessors on the level of $j(p)$, so again, because $\mathbb{P}$ is $N 5$-omitting, $r_{s} \perp r_{s^{\prime}}$.

Using Remark 4.10 and Proposition 3.11, by replacing $r_{s}$ and $r_{s^{\prime}}$ with proper successors, if necessary, we guarantee $r_{s} \perp_{s t} r_{s^{\prime}}$.

Any extension of $j$ that sends the elements of each $[q]_{p r} \subseteq \mathbb{Q}_{n+1}$ to distinct extensions of $r_{\operatorname{Pred}_{n}(q)}$ will be an embedding of $\mathbb{Q} \leqslant n+1$ into $\mathbb{P}$. It remains to see how to extend $j$ to an extendible embedding of $\mathbb{Q} \leqslant n+1$.

2. For each $[q] \subseteq \mathbb{Q}_{n+1}$, let $s=\operatorname{Pred}_{n}(q)$, and choose $r_{[q]} \geqslant_{\mathbb{P}} r_{s}$, such that if $\left[q^{\prime}\right] \neq[q]$ then $r_{\left[q^{\prime}\right]} \perp_{s t} r_{[q]}$. This is possible because every point in $\mathbb{P}$ has infinitely many incompatible successors, and successors of incompatible points are strongly incompatible. 
Any extension of $j$ that sends the elements of each $[q]$ to distinct $\equiv$-equivalent extensions of $r_{[q]}$ will satisfy conditions (1) and (2b) of the definition of $n$-extendible. (Satisfying (2b) makes sense, as (2b) depends only on $k_{[q]}$ for $q \in \mathbb{Q}_{\leqslant n}$.) It remains only to see how to define $\bar{j}$ and $\bar{k}$ on $\mathbb{Q}_{n+1}$ so condition (2a) is satisfied.

3. By assumption, above each $r_{[q]}$ is some $p$ such that $G_{[q]} \hookrightarrow G_{[p]}$. Choose such a $p$ and an embedding $\bar{k}_{[q]}: G_{[q]} \rightarrow G_{[p]}$.

4. For each $[q] \subseteq \mathbb{Q}_{n+1}$ and $q^{\prime} \in[q]$, let $\bar{j}\left(q^{\prime}\right)=\bar{k}_{[q]}\left(q^{\prime}\right)$.

This completes the construction.

Corollary 4.13. If $\mathbb{Q}$ is weakly proto-Ramsey, and $\mathbb{P}$ is proto-Ramsey and has incomparable points, then $\mathbb{Q} \hookrightarrow \mathbb{P}$ if and only if $\mathcal{G}(\mathbb{Q}) \hookrightarrow \mathcal{G}(\mathbb{P})$, if and only if $\mathcal{C} \ell \mathcal{G}(\mathbb{Q}) \subseteq \mathcal{C} \ell \mathcal{G}(\mathbb{P})$.

If $\mathbb{Q}$ and $\mathbb{P}$ are proto-Ramsey and have incomparable points, then $\mathbb{Q} \rightleftarrows \mathbb{P}$ if and only if $\mathcal{G}(\mathbb{Q}) \rightleftarrows \mathcal{G}(\mathbb{P})$, if and only if $\mathcal{C} \ell \mathcal{G}(\mathbb{Q})=\mathcal{C} \ell \mathcal{G}(\mathbb{P})$.

\subsection{Proving chain-Ramseyness from edge-Ramseyness and the joint embedding property}

Now we wish to show that if $\mathbb{P}$ is proto-Ramsey and has incompatible points, and $\mathcal{G}(\mathbb{P})$ is j.e.p. and edge-Ramsey, then $\mathbb{P}$ is chain-Ramsey. (We will handle the case where $\mathbb{P}$ does not have incompatible points later, in Proposition 4.20.) We will first show that if $c$ is a graph-induced coloring of $\mathbb{P}$, then $\mathbb{P}$ has a monochromatic isomorphic subordering. Since all colorings of singletons are graph-induced, this shows $R^{1}(\mathbb{P})$. Then, as the inductive step, we will show that if $R^{m}(\mathbb{P})$ holds, and $c$ is a coloring of $(m+1)$-chains from $\mathbb{P}$ in finitely many colors, then $\mathbb{P}$ has an isomorphic subordering on which $c$ is graph-induced. This shows that $R^{m}(\mathbb{P})$ implies $R^{m+1}(\mathbb{P})$, completing the proof.

Thus we must prove the following two propositions. In their proofs, we will build embeddings of $\mathbb{P}$ into itself. We will in each case indicate how to suitably modify the construction of an embedding in the proof of Proposition 4.11.

Proposition 4.14. If $\mathbb{P}$ is proto-Ramsey and has incompatible points, $\mathcal{G}(\mathbb{P})$ is j.e.p. and edge-Ramsey, and $c$ is a graph-induced coloring of $\mathbb{P}$, then there is an embedding of $\mathbb{P}$ into $\mathbb{P}$ whose range is monochromatic.

Proposition 4.15. If $\mathbb{P}$ is proto-Ramsey and has incompatible points, $R^{m}(\mathbb{P})$ holds, and $c$ is any coloring of $(m+1)$-chains in $\mathbb{P}$, then there is an embedding of $\mathbb{P}$ into $\mathbb{P}$ on whose range $c$ is graph-induced.

Now we prove Propositions 4.14 and 4.15.

The proof of Proposition 4.14 basically consists of constructing an embedding $j$ as in Proposition 4.11, guaranteeing that the range of $j$ is monochromatic by guaranteeing that, for some $i$, the range of every $k_{[q]}$ is monochromatic for $c_{[q]}$ in color $i$. (Recall that the $k_{[q]}$ are the graph embeddings of the $G_{[q]}$ into $G_{[j(q)]}$ for $q \in \mathbb{Q}_{n}$ that are used to determine the definition of $j$ on $\mathbb{Q}_{n+1}$.) This suffices by Proposition 4.7. 
To do this, we need Lemma 4.16, which shows that we can define $j$ on the minimal node of $\mathbb{P}$ and choose $i$ in such a way that we can always define each $k_{[q]}$ to have range monochromatic in color $i$. This, of course, uses the edge-Ramsey property of $\mathcal{G}(\mathbb{P})$ to guarantee the existence of monochromatic subgraphs.

Lemma 4.16. Suppose that $\mathbb{P}$ is proto-Ramsey, $\mathcal{G}(\mathbb{P})$ is j.e.p. and edge-Ramsey, and $c$ is a graph-induced coloring of $\mathbb{P}$ in $k$ colors $\{0,1, \ldots, k-1\}$. We identify $c$ with the corresponding collection of edge colorings on graphs $G_{a}$.

Then there is a color $i$ and there is an $r \in \mathbb{P}$ such that, for each $G_{a} \in \mathcal{G}(\mathbb{P})$, the set of $p$ such that $G_{[p]}$ contains a color $i$ monochromatic copy of $G_{a}$ is dense in $\mathbb{P}$ above $r$. That is, for any $q \geqslant_{\mathbb{P}} r$, there is $p \geqslant_{\mathbb{P}} q$ such that $G_{[p]}$ contains a color $i$ monochromatic copy of $G_{a}$.

Proof. Suppose not. Then for each $i$ and each $r \in \mathbb{P}$, there are a $G_{a} \in \mathcal{G}(\mathbb{P})$ and a $q \geqslant r$ such that for no $p \geqslant q$ does $G_{[p]}$ contain a color $i$ monochromatic copy of $G_{a}$.

Define a sequence as follows: $r_{0}$ is any element of $\mathbb{P}$. Given $r_{i}$, choose $a_{i}$ and $r_{i+1} \geqslant r_{i}$ such that for no $p \geqslant r_{i+1}$ does $G_{[p]}$ contain a color $i$ monochromatic copy of $G_{a_{i}}$. Then, for no color $i<k$ and no $p \geqslant r_{k}$ does $G_{[p]}$ contain a color $i$ monochromatic copy of $G_{a_{i}}$. Because $\mathcal{G}(\mathbb{P})$ has the joint embedding property, there is some $\equiv$ equivalence class $a$ such that, for all $i<k$, we have $G_{a_{i}} \hookrightarrow G_{a}$. But then for no $p \geqslant r_{k}$ does $G_{[p]}$ contain a monochromatic copy of $G_{a}$ in any color, contradicting our assumptions that $\mathcal{G}(\mathbb{P})$ is edge-Ramsey and $\mathbb{P}$ is densely self-embeddable.

Proof of Proposition 4.14. Suppose $c$ is a graph-induced coloring. We must find an embedding of $\mathbb{P}$ into $\mathbb{P}$ with monochromatic image.

Choose $i$ and $r$ as in Lemma 4.16, such that for any $q \geqslant_{\mathbb{P}} r$, there is $p \geqslant_{\mathbb{P}} q$ such that $G_{[p]}$ contains a color $i$ monochromatic copy of $G_{a}$.

We construct an embedding of $\mathbb{P}$ into $\mathbb{P}$ as the limit of a sequence of extendible $n$ embeddings, as in the proof of Proposition 4.11, guaranteeing at each stage that all edges in the image have color $i$.

Begin by defining an extendible 0-embedding $j$ as follows. Choose $p \geqslant_{\mathbb{P}} r$ connected by an edge of color $i$ to $t \in S\left(G_{[p]}\right)$. Set $k_{\left[q_{0}\right]}\left(q_{0}\right)=j\left(q_{0}\right)=p$ and, for the unique element $s$ of $S\left(G_{\left[q_{0}\right]}\right)$, set $k_{\left[q_{0}\right]}(s)=t$.

Extend each extendible $n$-embedding to an extendible $n+1$-embedding using the construction of Proposition 4.11. In step (3), choose $p$ and $\bar{k}_{[q]}: G_{[q]} \rightarrow G_{[p]}$ such that the range of $\bar{k}_{[q]}$ is monochromatic in color $i$. This is possible by the choice of $i$ and $r$.

By Proposition 4.7, the range of the embedding thus constructed is monochromatic for $c$ in color $i$.

Corollary 4.17. If $\mathbb{P}$ is proto-Ramsey and $\mathcal{G}(\mathbb{P})$ is j.e.p. and edge-Ramsey, then $R^{1}(\mathbb{P})$.

Proof. Any coloring of singletons is graph-induced.

Now we prove Proposition 4.15, which states that if $R^{m}(\mathbb{P})$ holds and if $c$ is a coloring of $m+1$-chains from $\mathbb{P}$, then there is an isomorphic suborder on which $c$ is graph-induced. 
To explain the idea behind this proof, we consider the simpler result that if $\mathbb{P}$ is the complete binary tree, then $R^{1}(\mathbb{P}) \Longrightarrow R^{2}(\mathbb{P})$. Given a coloring $c$ of 2 -chains, we build an embedding $j$ on which the color of a chain $\{p, q\}$ depends only on the first element $p$, as follows. The minimal element of $\mathbb{P}$ is \langle\rangle : choose $j(\langle\rangle)$ arbitrarily. Now choose a copy of the binary tree $\mathbb{Q}$ above $j(\langle\rangle)$ and a color $d_{\langle\rangle}$such that for all $q \in \mathbb{Q}$, we have $c(j(\langle\rangle), q)=d_{\langle\rangle}$; we can do this by $R^{1}(\mathbb{P})$. Choose incomparable points $j(\langle 0\rangle)=q_{0}$ and $j(\langle 1\rangle)=q_{1}$ from $\mathbb{Q}$. Now, choose copies of the binary tree $\mathbb{Q}_{i} \subseteq \mathbb{Q}$ above $j(\langle i\rangle)$ and colors $d_{\langle i\rangle}$ such that for all $q \in \mathbb{Q}_{\langle i\rangle}$, we have $c(j(\langle i\rangle), q)=d_{\langle i\rangle}$. Iterate this process, at each stage choosing values $j(p)$ inside the previously chosen monochromatic subtrees. By construction, for any 2 -chain $j(p)<_{\mathbb{P}} q$ in the image of $j$, we have $c(j(p), q)=d_{p}$.

For a more complex $\mathbb{P}$, we carry out the analogous construction. In general, this produces not a coloring of 2-chains that depends only on the smaller element, but a coloring of 2-chains that is graph-induced. To see this, consider the analogous case where we have defined $j(p)$ and now must define $j(r)$ and $j\left(r^{\prime}\right)$ for $r$ and $r^{\prime}$ above $p$ on the next level of $\mathbb{P}$. If $r$ and $r^{\prime}$ have different predecessor sets on the level of $p$, say $s$ and $s^{\prime}$, then we must choose $j(r)$ above $k(s)$ and $j\left(r^{\prime}\right)$ above $k\left(s^{\prime}\right)$. We can choose these points in suborderings above $k(s)$ and $k\left(s^{\prime}\right)$ monochromatic for the coloring $\bar{c}(q)=c(j(p), q)$, but those suborderings may not be monochromatic for the same color. Hence, the color will depend not only on $p$ but also on $s$. That is, on the image of $j$, the coloring will be graph-induced.

This proof uses the inductive hypothesis $R^{m}(\mathbb{P})$, but does not use the edge-Ramseyness of $\mathcal{G}(\mathbb{P})$.

Proof of Proposition 4.15. Suppose $c$ is a coloring of $m+1$-chains in $\mathbb{P}$, and $R^{m}(\mathbb{P})$ holds. We must find an embedding $j: \mathbb{P} \rightarrow \mathbb{P}$ on whose range $c$ is graph-induced.

We define an extendible $n$-embedding $j$ to be color-extendible if there are functions $k_{[u]}$ witnessing that $j$ is extendible and, for each $s \in S\left(G_{[u]}\right)$ for $u \in \mathbb{P}_{\leqslant n}$, a substructure $\mathbb{P}_{s} \cong \mathbb{P}$ with least element $p_{s}$ such that:

(3.) $\operatorname{Pred}_{\operatorname{lev}(j(u))}\left(p_{s}\right)=k_{[u]}(s)$.

(4.) For all $p \in s$, there is a color $i_{p, s}$ such that, for all $\left\langle j(p), p_{2}, p_{3}, \ldots, p_{m+1}\right\rangle$ from range $(j) \cup \mathbb{P}_{s}$, we have $c\left(j(p), p_{2}, p_{3}, \ldots, p_{m+1}\right)=i_{p, s}$.

(5.) If $q \in \mathbb{P}_{\leqslant n}, s=\operatorname{Pred}_{m}(q)$, and $q \in s^{\prime} \in S\left(G_{[q]}\right)$, then $j(q) \in \mathbb{P}_{s}$ and $\mathbb{P}_{s^{\prime}} \subseteq \mathbb{P}_{s}$.

We show that every color-extendible $n$-embedding can be extended to a color-extendible $(n+1)$-embedding. Then we embed $\mathbb{P}$ into $\mathbb{P}$ via the limit of a sequence of colorextendible $n$-embeddings. On the image, $c$ is graph-induced.

First we show there is a color-extendible 0-embedding. Let $q_{0}$ be the least element of $\mathbb{P}$. Choose $j\left(q_{0}\right)=k_{\left[q_{0}\right]}\left(q_{0}\right)$ arbitrarily. Because $\mathbb{P}$ is densely self-embeddable, there is a copy $\mathbb{Q}$ of $\mathbb{P}$ above $j\left(q_{0}\right)$. We can color $m$-chains from that copy by $\bar{c}\left(z_{2}, \ldots, z_{m+1}\right)=$ $c\left(j\left(q_{0}\right), z_{2}, \ldots, z_{m+1}\right)$. Choose a $\bar{c}$-monochromatic in color $i$ copy $\overline{\mathbb{Q}} \subseteq \mathbb{Q}$ with least element $z$. For the unique element $s$ of $S\left(G_{\left[q_{0}\right]}\right)$, set $k_{\left[q_{0}\right]}(s)=\operatorname{Pred}_{l e v\left(j\left(q_{0}\right)\right)}(z)$ and $\mathbb{P}_{s}=\overline{\mathbb{Q}}$ and $i_{q_{0}, s}=i$.

Now we extend a color-extendible $n$-embedding $j$ with witnesses $\left\{k_{[u]} \mid[u] \subseteq \mathbb{P}_{n}\right\}$ and $\left\{\mathbb{P}_{s} \mid s \subseteq[u]\right\}$ to a color-extendible $n+1$-embedding $\bar{j}$. We follow the construction of the 
proof of Proposition 4.11, working inside the $\mathbb{P}_{s}$. More precisely:

In step (1), take $r_{s}>_{\mathbb{P}} p_{s}$ with $r_{s}$ in $\mathbb{P}_{s}$.

In step (2), for $[q] \subseteq s=[q]_{p r}$, choose $r_{[q]} \in \mathbb{P}_{s}$.

In step (3), choose $G_{[p]}$ in $\mathbb{P}_{s}$. More precisely, for $p \in \mathbb{P}_{s}$, let $\bar{G}_{[p]}$ denote $G_{[p]}$ as defined in $\mathbb{P}_{s}$. Choose $p \in \mathbb{P}_{s}$ with $\bar{k}: G_{[q]} \rightarrow \bar{G}_{[p]}$. By Proposition 4.7, the inclusion map from $\mathbb{P}_{s}$ to $\mathbb{P}$ induces an embedding $i: \bar{G}_{[p]} \rightarrow G_{[p]}$. Define $\bar{k}_{[q]}: G_{[q]} \rightarrow G_{[p]}$ by $\bar{k}_{[q]}=i \circ \bar{k}$.

Having defined $\bar{j}(q)$ and $\bar{k}_{[q]}$ for $q \in \mathbb{P}_{n+1}$, we must define $\mathbb{P}_{\bar{s}}$ for $\bar{s} \in S\left(G_{[q]}\right)$.

Suppose $\operatorname{Pred}_{n}(q)=s$. We need to choose $\mathbb{P}_{\bar{s}} \subseteq \mathbb{P}_{s}$ to satisfy (4) with respect to some choice of $i_{p, \bar{s}}$ for $p \in \mathbb{P}_{n+1}$.

To do this, for $\left.\bar{s} \in S\left(G_{[q]}\right)\right)$, by our choice of $G_{[p]}$ in step (3), we have $k_{[q]}(\bar{s})=$ Pred $_{l e v(\bar{j}(q))}(r)$, for some $r \in \mathbb{P}_{s}$. Let $\mathbb{Q} \subseteq\left\{\bar{q} \in \mathbb{P}_{s} \mid r \leqslant \mathbb{P} \bar{q}\right\}$ be isomorphic to $\mathbb{P}$ and color $m$-chains from $\mathbb{Q}$ by

$$
\bar{c}\left(p_{2}, \ldots, p_{m+1}\right)=\left\langle c\left(j(p), p_{2}, \ldots, p_{m+1}\right) \mid p \in \bar{s}\right\rangle .
$$

Since $\bar{s}$ is finite, $\bar{c}$ is a coloring in finitely many colors. Choose $\mathbb{P}_{\bar{s}} \subseteq \mathbb{Q}$ to be monochromatic with color $\left\langle c_{p} \mid p \in \bar{s}\right\rangle$ and define $i_{p, \bar{s}}=c_{p}$.

This completes the construction.

For all chains $\left\langle j(p), p_{2}, p_{3}, \ldots, p_{m+1}\right\rangle$ from $\operatorname{range}(j)$, by construction, $\left\{p_{2}, p_{3}, \ldots, p_{m+1}\right\}$ $\subset \mathbb{P}_{s}$ where $s=\operatorname{Pred}_{l e v(p)}(r)$ for $r$ such that $j(r)=p_{2}$, and so $c\left(j(p), p_{2}, p_{3}, \ldots, p_{m+1}\right)=$ $i_{p, s}$. Thus, $c\left(j(p), p_{2}, p_{3}, \ldots, p_{m+1}\right)$ depends only on $p$ and $s$. Since $p$ is determined by $j(p)$ and $s$ is determined by $k_{[p]}(s)=\operatorname{Pred}_{l e v(j(p))}\left(p_{2}\right), c\left(j(p), p_{2}, p_{3}, \ldots, p_{m+1}\right)$ depends only on $j(p)$ and $\operatorname{Pred}_{l e v(j(p))}\left(p_{2}\right)$. That is, on the range of $j$, the coloring $c$ is graph-induced.

\subsection{The singular case}

Now we consider the singular case in which every graph $G \in \mathcal{G}(\mathbb{P})$ has only one element in $S(G)$, and $\mathbb{P}$ may or may not contain incompatible points. In this case, if incomparable elements of $\mathbb{P}$ are compatible, then they have exactly the same successors in $\mathbb{P}$. This case is anomalous because, by Corollary 4.13 , it is only in this case that for non-biembeddable proto-Ramsey partial orderings $\mathbb{P}$ and $\mathbb{Q}$ we may have $\mathcal{C} \ell(\mathcal{G}(\mathbb{P}))=\mathcal{C} \ell(\mathcal{G}(\mathbb{Q}))$.

In order to analyze the singular case, we first make the following definitions.

Definition 4.18. For $\mathbb{P}$ an $\omega$-linearizable partial ordering and $n<\omega$,

$$
\begin{gathered}
\operatorname{Mult}_{n}(\mathbb{P})=\{\langle p, i\rangle \mid p \in \mathbb{P} \& i<n\} \\
\operatorname{Mult}(\mathbb{P})=\{\langle p, i\rangle \mid p \in \mathbb{P} \& i \leqslant \operatorname{lev}(p)\}
\end{gathered}
$$

in both cases ordered by

$$
\langle p, i\rangle<\langle q, j\rangle \Longleftrightarrow p<\mathbb{P} q .
$$

Definition 4.19. The complete bipartite graph $G$ with a single point in $S(G)$ and $n$ points in $M(G)$ is denoted $\operatorname{Sing}(n)$. Thus $\operatorname{Sing}(1)$ is the graph with two vertices connected by a single edge. 
Proposition 4.20. Suppose that $\mathbb{P}$ is proto-Ramsey and singular (every graph $G \in \mathcal{G}(\mathbb{P})$ has only one element in $S(G)$ ). Then there are three possible cases.

1. If $\mathcal{G}(\mathbb{P})$ contains only Sing $(1)$, then $\mathbb{P}$ is biembeddable with either $\omega$ or $2^{<\omega}$. The collection $\mathcal{G}(\mathbb{P})$ is edge-Ramsey and has the joint embedding property, and the partial ordering $\mathbb{P}$ is chain-Ramsey.

2. If $\mathcal{G}(\mathbb{P})$ contains Sing $(n)$ for arbitrarily large $n$, then $\mathbb{P}$ is biembeddable with either Mult $(\omega)$ or $\operatorname{Mult}\left(2^{<\omega}\right)$. The collection $\mathcal{G}(\mathbb{P})$ is edge-Ramsey and has the joint embedding property, and the partial ordering $\mathbb{P}$ is chain-Ramsey.

3. If $\mathcal{G}(\mathbb{P})$ contains Sing $(n)$ for some largest $n>1$, then $\mathbb{P}$ is biembeddable with either Mult $_{n}(\omega)$ or Mult ${ }_{n}\left(2^{<\omega}\right)$. The collection $\mathcal{G}(\mathbb{P})$ is not edge-Ramsey and the partial ordering $\mathbb{P}$ is not chain-Ramsey.

Proof. If $\mathbb{P}$ has incompatible points, then by Corollary $4.13 \mathbb{P}$ is biembeddable with $2^{<\omega}$, $\operatorname{Mult}\left(2^{<\omega}\right)$, or $\operatorname{Mult}_{n}\left(2^{<\omega}\right)$, in cases (1), (2), and (3), respectively. If not, then $\mathbb{P}$ has finitely many points on each level, and points on different levels are always comparable, in which case the biembeddability class of $\mathbb{P}$ is determined by the maximum number $n$ of points on any level, or the lack of such a maximum. This is because if there is one level with at least $n$-many points, then because $\mathbb{P}$ is densely self-embeddable, there are infinitely many such levels.

In case $(3)$, it is easy to see that $\mathcal{G}(\mathbb{P})$ is not edge-Ramsey, and so by Proposition 4.8 , $\mathbb{P}$ is not chain-Ramsey.

In cases $(1)$ and $(2), \mathbb{P}$ is chain-Ramsey [11],[1],[2]. Thus, by Proposition 4.8, $\mathcal{G}(\mathbb{P})$ is edge-Ramsey and has the joint embedding property. This is also easy to check directly.

\subsection{Characterization theorems}

We have finished proving the following theorem.

Theorem 4.21. Let $\mathbb{P}$ be a proto-Ramsey partial ordering. Then $\mathbb{P}$ is chain-Ramsey if and only if $\mathcal{G}(\mathbb{P})$ is edge-Ramsey and has the joint embedding property.

Proof. The $\Longrightarrow$ direction is Proposition 4.8 , and the $\Longleftarrow$ direction follows from Propositions 4.14 and 4.15 for $\mathbb{P}$ with incompatible elements, and from Proposition 4.20 for $\mathbb{P}$ without incompatible elements.

Corollary 4.22. Let $\mathbb{P}$ be a finite-level partial ordering. Then $\mathbb{P}$ is chain-Ramsey if and only if

(a.) $\mathbb{P}$ is biembeddable with a proto-Ramsey partial ordering, and

(b.) $\mathcal{G}(\mathbb{P})$ is edge-Ramsey and has the joint-embedding property.

Now we address a question raised earlier.

Proposition 4.23. If $\mathbb{P}$ is a countably infinite partial ordering and $\mathbb{P} \rightarrow(\mathbb{P})^{A}$ for all finite partial orderings $A$, then $\mathbb{P}$ is biembeddable with one of the following: an infinite antichain, $\omega, \operatorname{Mult}(\omega), \omega^{*}$, or $(\operatorname{Mult}(\omega))^{*}$. 
Proof. By way of contradiction, suppose that $\mathbb{P}$ is a counterexample. By Proposition 3.1, we may assume that elements of $\mathbb{P}$ are natural numbers, and that $p<\mathbb{P} q \Longrightarrow p<q$.

First, let $B$ be the partial ordering consisting of four points $a_{1}<a_{2}$ and $b_{1}<b_{2}$ with $a_{i}$ incomparable with $b_{j}$. We will show that $\mathbb{P}$ cannot contain a copy of $B$. Let $A$ be the partial ordering consisting of three points, $c_{1}<c_{2}$, and $d$ incomparable to both $c_{1}$ and $c_{2}$.
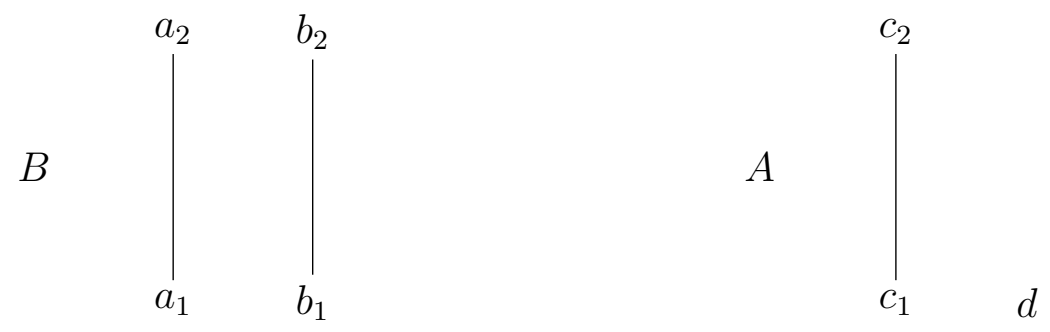

Color a copy $p_{1}<_{\mathbb{P}} p_{2}, q$ of $A$ in $\mathbb{P}$ with color 0 if $p_{1}<q$ (as natural numbers), and with color 1 if $q<p_{1}$. Replace $\mathbb{P}$ with a monochromatic suborder. Now, in $\mathbb{P}$ we cannot have a copy $p_{1}<\mathbb{P} p_{2}, q_{1}<\mathbb{P} q_{2}$ of $B$, for suppose $p_{1}<q_{1}$ as natural numbers. Then $p_{1}, p_{2}, q_{1}$ is a copy of $A$ in color 0 , and $q_{1}, q_{2}, p_{1}$ is a copy of $A$ in color 1 , which is a contradiction.

If $\mathbb{P}$ is finite-level, then since $\mathbb{P}$ is chain-Ramsey, $\mathbb{P}$ is biembeddable with a protoRamsey partial ordering $\mathbb{Q}$. Now $\mathbb{Q}$ cannot have incompatible elements, or else it would contain a copy of $B$. Hence, by Proposition $4.20, \mathbb{P}$ must be biembeddable with either $\omega$ or $\operatorname{Mult}(\omega)$, and thus not a counterexample.

Hence, $\mathbb{P}$ is not finite-level, so $\mathbb{P}$ has an infinite antichain. Color pairs of incomparable points in $\mathbb{P}$ color 0 if they are on different levels, and color 1 if they are on the same level. Let $\mathbb{Q}$ be a monochromatic suborder.

First, suppose $\mathbb{Q}$ has color 1 . Then $\mathbb{Q}$ has an infinite antichain $X \subseteq \mathbb{P}_{n}$ for some $n$, and all incomparable pairs in $\mathbb{Q}$ must be contained in $X$. No element of $\mathbb{Q}$ can be in $\mathbb{P}_{m}$ for any $m>n$, because such an element would be comparable to (hence greater than) all elements of $X$, contradicting $p<\mathbb{P} q \Longrightarrow p<q$. Now if we color elements of $\mathbb{Q}$ with $n+1$ colors by $c(p)=\operatorname{lev}(p)$, a monochromatic suborder must be an antichain. Therefore $\mathbb{P}$ must in fact consist of a single antichain, so $\mathbb{P}$ is not a counterexample.

Now suppose $\mathbb{Q}$ has color 0 , and let $X$ be an infinite antichain, so elements of $X$ are on arbitrarily high levels of $\mathbb{P}$. All elements of $X$ must be maximal elements of $\mathbb{P}$. (Suppose not. Then consider some element $p \in X$ with a successor $p^{\prime}$ in $\mathbb{P}$. Consider another element $q \in X$ at least two levels above the level of $p^{\prime}$, and an immediate predecessor $q^{\prime}$ of $q$ in $\mathbb{P}$. Then $\left\{p, p^{\prime}, q^{\prime}, q\right\}$ is a copy of $B$.) Now color elements of $\mathbb{Q}$ color 0 if they are maximal in $\mathbb{P}$, and color 1 otherwise. A monochromatic suborder of color 1 cannot be isomorphic to $\mathbb{P}$. (A monochromatic suborder of color 1 cannot have an infinite antichain, because by the same argument we applied to $\mathbb{Q}$, an infinite antichain must consist of elements maximal in $\mathbb{P}$.) A monochromatic isomorphic suborder, then, must have color 0 so, since it consists entirely of elements maximal in $\mathbb{P}$, it must be an antichain. Again, $\mathbb{P}$ must in fact consist of a single antichain, so $\mathbb{P}$ is not a counterexample. 


\section{Canonical Representatives}

In this section we specify a canonical representative of each biembeddability class of finitelevel proto-Ramsey partial orderings.

The singular cases where $\mathcal{G}(\mathbb{P}) \subseteq\{\operatorname{Sing}(n) \mid n \in \omega\}$ are covered by Proposition 4.20; the canonical representatives are $\omega, 2^{<\omega}, \operatorname{Mult}_{n}(\omega), \operatorname{Mult}_{n}\left(2^{<\omega}\right), \operatorname{Mult}(\omega)$ and $\operatorname{Mult}\left(2^{<\omega}\right)$. The following construction addresses other cases.

\subsection{Constructing $\mathbb{P}$ from $\mathcal{G}$}

Definition 5.1. A bipartite graph $G$ is of finite down-degree if every point in $S(G)$ is connected by edges to only finitely many points of $M(G)$.

If $G \in \mathcal{G}(\mathbb{P})$ for some weakly proto-Ramsey $\mathbb{P}$ then, by $\omega$-linearizability, $G$ must be of finite down-degree.

Definition 5.2. Let $\overrightarrow{\mathcal{G}}=\left\langle G_{n} \mid n<\omega\right\rangle$ be any sequence of finite or countable connected extensional bipartite graphs of finite down-degree, with $G_{0}=\operatorname{Sing}(1)$. Define a weakly proto-Ramsey partial ordering $\mathbb{P}(\overrightarrow{\mathcal{G}})$, level by level, as follows:

Stage 0: At level 0 put a single point $p_{0}$, to correspond to the single point of $M\left(G_{0}\right)$. At level 1, put a single equivalence class, to correspond to the single point of $S\left(G_{0}\right)$; all elements of this equivalence class will be above the single point at level 0 . At stage 1 we will determine the elements of this equivalence class. Stage 0 guarantees that $G_{\left[p_{0}\right]} \cong G_{0}$.

Stage $n$ for $n>0$ : At stage $n-1$ we determined which equivalence classes would be on level $n$, and which points on level $n-1$ they would lie above. For each equivalence class $a$, set $a=\left\{p_{x, a} \mid x \in M\left(G_{n}\right)\right\}$. Now for each equivalence class $a$ and each $y \in S\left(G_{n}\right)$, put on level $n+1$ an equivalence class $c(a, y)$, lying above exactly the points $\left\{p_{x, a} \in a \mid\langle x, y\rangle \in\right.$ $\left.E\left(G_{n}\right)\right\}$. Stage $n$ guarantees that $G_{[q]} \cong G_{n}$ for every $q$ on level $n$ of $\mathbb{P}(\overrightarrow{\mathcal{G}})$.

The partial ordering is determined by closing the relation between levels to make it transitive.

Proposition 5.3. If $\overrightarrow{\mathcal{G}}=\left\langle G_{n} \mid n<\omega\right\rangle$ is any sequence of finite or countable connected extensional bipartite graphs of finite down-degree with $G_{0}=\operatorname{Sing}(1)$, then $\mathbb{P}(\overrightarrow{\mathcal{G}})$ is weakly proto-Ramsey, and $\mathcal{C} \ell(\mathcal{G}(\mathbb{P}(\overrightarrow{\mathcal{G}})))=\mathcal{C} \ell\left(\left\{G_{n} \mid n \in \omega\right\}\right)$. Furthermore, if for every $m$ there is $n>m$ such that $G_{m} \hookrightarrow G_{n}$, then $\mathbb{P}(\overrightarrow{\mathcal{G}})$ is densely self-embeddable, hence proto-Ramsey, and if every $G_{n}$ is finite, then $\mathbb{P}(\overrightarrow{\mathcal{G}})$ is finite-level.

Proof. By construction, $\mathbb{P}(\overrightarrow{\mathcal{G}})$ has a least element. By induction, every level of $\mathbb{P}(\overrightarrow{\mathcal{G}})$ is countable (finite if each $G_{n}$ is finite), and every element has finitely many predecessors, so $\mathbb{P}(\overrightarrow{\mathcal{G}})$ is $\omega$-linearizable (and finite-level if each $G_{n}$ is finite).

By construction, two elements on the same level of $\mathbb{P}(\overrightarrow{\mathcal{G}})$ have a common successor on the next level only if they have the same predecessors (as they must be in the same equivalence class). To show $\mathbb{P}(\overrightarrow{\mathcal{G}})$ is $N 5$-omitting, hence weakly proto-Ramsey, it suffices to show that any pair of incomparable elements $p$ and $q$ with any common successor have 
the same predecessors. To show this, suppose $r$ is a common successor of $p$ and $q$ of minimal level, say level $n+1$. Because the partial ordering is the transitive closure of the ordering between levels, there are $\bar{p}$ and $\bar{q}$ on level $n$ such that $p \leqslant_{\mathbb{P}(\overrightarrow{\mathcal{G}})} \bar{p} \leqslant_{\mathbb{P}(\overrightarrow{\mathcal{G}})} r$ and $q \leqslant_{\mathbb{P}(\overrightarrow{\mathcal{G}})} \bar{q} \leqslant_{\mathbb{P}(\overrightarrow{\mathcal{G}})} r$. Because $\bar{p}$ and $\bar{q}$ are on the same level and are compatible, they must have the same predecessors, including $p$ if $p<_{\mathbb{P}(\overrightarrow{\mathcal{G}})} \bar{p}$ and $q$ if $q<_{\mathbb{P}(\overrightarrow{\mathcal{G}})} \bar{q}$. By the minimality of the level of $r$, then, $p=\bar{p}$ and $q=\bar{q}$, so $p$ and $q$ have the same predecessors.

By construction, as for $q \in \mathbb{P}(\mathcal{G})_{n}$ we have $G_{[q]} \cong G_{n}$, we have $\mathcal{C} \ell(\mathcal{G}(\mathbb{P}(\overrightarrow{\mathcal{G}})))=$ $\mathcal{C} \ell\left(\left\{G_{n} \mid n \in \omega\right\}\right)$.

If for every $m$ there is $n>m$ such that $G_{m} \hookrightarrow G_{n}$, then by Proposition 4.11 in the nonsingular case, and by an easy argument in the singular case, $\mathbb{P}(\overrightarrow{\mathcal{G}})$ is densely self-embeddable, hence proto-Ramsey.

Definition 5.4. Fix an enumeration of the collection of all nontrivial connected extensional finite bipartite graphs $G$ with $M(G) \subset \omega$ and $S(G) \subset \omega$, with a copy of the single-edge graph listed first.

If $\mathcal{G}$ is any collection of nontrivial connected extensional finite bipartite graphs, let $\mathcal{C} \ell(\mathcal{G})$ be the induced enumeration of $\mathcal{C} \ell(\mathcal{G})$, and define $\mathbb{P}(\mathcal{G})=\mathbb{P}(\mathcal{C} \ell(\mathcal{G}))=\mathbb{P}(\mathcal{C} \ell(\mathcal{G}))$.

Proposition 5.5. If $\mathbb{P}$ is a finite-level proto-Ramsey partial ordering such that at least one $G_{a} \in \mathcal{G}(\mathbb{P})$ has more than one point in $S\left(G_{a}\right)$, then $\mathbb{P} \rightleftarrows \mathbb{P}(\mathcal{G}(\mathbb{P}))$.

Furthermore, if $\mathcal{G}$ is any collection of nontrivial connected extensional finite bipartite graphs, then $\mathcal{C} \ell(\mathcal{G}(\mathbb{P}(\mathcal{G})))=\mathcal{C} \ell(\mathcal{G})$, and $\mathbb{P}(\mathcal{C} \ell(\mathcal{G}))$ is proto-Ramsey.

Proof. Note that each $G \in \mathcal{G}$ has infinitely many isomorphic copies in $\mathcal{C} \ell \mathcal{G}$, so $\mathcal{C} \vec{\ell}$ has the property that for every $m$ there is $n>m$ such that $G_{m} \hookrightarrow G_{n}$.

The first part of Proposition 5.5 follows from Propositions 5.3 and 4.11. The second follows directly from Proposition 5.3.

\subsection{Summary of results}

We have shown the following:

If $\mathbb{P}$ is a finite-level or weakly proto-Ramsey partial ordering, and if $\mathbb{P}$ is chain-Ramsey, then by Propositions 3.8 and $3.10, \mathbb{P}$ is biembeddable with a proto-Ramsey partial ordering.

(In general, by Proposition 4.11, a nonsingular weakly proto-Ramsey partial ordering $\mathbb{P}$ is isomorphic to a subordering of $\mathbb{P}(\mathcal{G}(\mathbb{P}))$. A Cantor-Bendixson-type analysis determines whether $\mathbb{P}$ contains a copy of $\mathbb{P}(\mathcal{G}(\mathbb{P}))$, an analogue of a perfect kernel. If $\mathbb{P}$ is chainRamsey, by Propositions 3.8, 3.10, 4.7 and 4.11, it must contain such a copy. A similar analysis holds in the singular case, with $\mathbb{P}(\mathcal{G}(\mathbb{P})$ replaced by one of the partial orderings of Proposition 4.20.)

If $\mathbb{P}$ is a proto-Ramsey partial ordering, then by Theorem $4.21, \mathbb{P}$ is chain-Ramsey if and only if $\mathcal{G}(\mathbb{P})$ is edge-Ramsey and has the joint embedding property.

Furthermore, if $\mathbb{P}$ is finite-level, proto-Ramsey and nonsingular, then, by Corollary 4.13 and Proposition 5.5, there is a canonical representative $\mathbb{P}(\mathcal{G}(\mathbb{P}))$ of the biembeddability 
class of $\mathbb{P}$. In the nonsingular case, by Proposition 5.5, there is a one-to-one correspondence between biembeddabilty classs of proto-Ramsey partial orderings and collections of finite bipartite graphs of the form $\mathcal{C} \ell \mathcal{G}$, associating the class of $\mathbb{P}$ to the collection $\mathcal{C} \ell(\mathcal{G}(\mathbb{P})$ ) and the collection $\mathcal{C} \ell \mathcal{G}$ to the class of $\mathbb{P}(\mathcal{G})$. In the singular case, each collection $\mathcal{C} \ell \mathcal{G}$ corresponds to two classes of partial orderings, one with branching and one without. Canonical representatives of classes of singular proto-Ramsey partial orderings are given by Proposition 4.20 .

In general, Proposition 5.3 gives us a similar analysis for infinite-level partial orderings $\mathbb{P}$, with the complication that if $\mathcal{G}$ contains an infinite graph, then $\mathcal{C} \ell(\mathcal{G})$ is not countable. However, if $\mathcal{G}$ is any countable collection of connected, extensional, countable bipartite graphs of finite down-degree, then $\mathcal{C} \ell(\mathcal{G})$ corresponds to a proto-Ramsey partial ordering $\mathbb{P}$, constructed as follows. Choose a sequence $\left\langle G_{n} \mid n \in \omega\right\rangle$ from $\mathcal{C} \ell(\mathcal{G})$, such that for every $G \in \mathcal{G}$ there are infinitely many $n$ such that $G \hookrightarrow G_{n}$, so $\mathcal{C} \ell(\mathcal{G})=\mathcal{C} \ell\left(\left\{G_{n} \mid n \in \omega\right\}\right)$. Then $\mathcal{C} \ell\left(\mathcal{G}\left(\mathbb{P}\left(\left\langle G_{n} \mid n \in \omega\right\rangle\right)\right)\right)=\mathcal{C} \ell \mathcal{G}$. Since $\mathcal{G}(\mathbb{P})$ is in fact countable, applying this construction to $\mathcal{G}(\mathbb{P})$ produces a representative of the biembeddability class of $\mathbb{P}$, unique up to the choice of the sequence $\left\langle G_{n} \mid n \in \omega\right\rangle$.

In summary, the constructions $\mathcal{C} \ell(\mathcal{G}(\mathbb{P}))$ and $\mathbb{P}(\mathcal{G})$ provide almost one-to-one associations (the anomalous singular cases being covered by Proposition 4.20) of biembeddability classes of proto-Ramsey partial orderings with countably generated collections $\mathcal{C} \ell \mathcal{G}$ of connected extensional countable bipartite graphs of finite down-degree, by which chainRamsey partial orderings are associated with edge-Ramsey j.e.p. collections of graphs, and conversely.

\section{Generating Examples}

So far, the only explicit examples of countably infinite chain-Ramsey partial orderings we have seen are those biembeddable with $\omega, 2^{<\omega}, \operatorname{Mult}(\omega)$ or $\operatorname{Mult}\left(2^{<\omega}\right)$ (or with $\omega^{*}$, $\left(2^{<\omega}\right)^{*},(\operatorname{Mult}(\omega))^{*}$ or $\left.\left(\operatorname{Mult}\left(2^{<\omega}\right)\right)^{*}\right)$.

We have seen that finite-level chain-Ramsey partial orderings $\mathbb{P}$ correspond to edgeRamsey j.e.p. collections $\mathcal{G}(\mathbb{P})$ of finite bipartite graphs, and conversely. Hence, from families of finite bipartite graphs that have been shown to be edge-Ramsey, we can define new finite-level chain-Ramsey partial orderings.

In this section we note another way in which a chain-Ramsey partial ordering $\mathbb{P}$ generates an edge-Ramsey family of finite bipartite graphs, in general not the same as $\mathcal{G}(\mathbb{P})$. Using this family $\mathcal{G}$ to determine a new finite-level chain-Ramsey partial ordering $\mathbb{P}(\mathcal{G}) \not \subset \mathbb{P}$, and iterating, we can construct new examples.

Definition 6.1. For $\mathbb{P}$ a partial ordering, we define the following bipartite graphs:

The graph $G_{<}(\mathbb{P})=\left\langle\left\{x_{p} \mid p \in \mathbb{P}\right\},\left\{y_{q} \mid q \in \mathbb{P}\right\},\left\{\left\langle x_{p}, y_{q}\right\rangle \mid p<\mathbb{P} q\right\}\right\rangle$.

The graph $G_{>}(\mathbb{P})=\left\langle\left\{x_{p} \mid p \in \mathbb{P}\right\},\left\{y_{q} \mid q \in \mathbb{P}\right\},\left\{\left\langle x_{p}, y_{q}\right\rangle|p\rangle_{\mathbb{P}} q\right\}\right\rangle$.

We then define the following collections of finite bipartite graphs:

The collection of all finite subgraphs of $G_{<}(\mathbb{P})$, denoted $\mathcal{G}_{<}(\mathbb{P})$.

The collection of all finite subgraphs of $G_{>}(\mathbb{P})$, denoted $\mathcal{G}_{>}(\mathbb{P})$. 
Proposition 6.2. If $\mathbb{P}$ is a chain-Ramsey partial ordering, then $G_{<}(\mathbb{P})$ and $G_{>}(\mathbb{P})$ are edge-Ramsey bipartite graphs, and $\mathcal{G}_{<}(\mathbb{P})$ and $\mathcal{G}_{>}(\mathbb{P})$ are edge-Ramsey collections of finite bipartite graphs with the joint embedding property.

Proof. If $G$ is $G_{<}(\mathbb{P})$ [or $\left.G_{>}(\mathbb{P})\right]$, a coloring of edges of $G$ is exactly a coloring of 2-element chains from $\mathbb{P}$, so if $R^{2}(\mathbb{P})$ holds, we can find a monochromatic isomorphic subordering $\mathbb{Q}$. Restricting the points of $G$ to points $x_{q}$ and $y_{q}$ for $q \in \mathbb{Q}$ produces, then, a monochromatic isomorphic subgraph. Hence $G$ is edge-Ramsey.

Now $\mathcal{G}_{<}(\mathbb{P})\left[\right.$ or $\left.\mathcal{G}_{>}(\mathbb{P})\right]$ is the collection of finite subgraphs of $G$, which is edge-Ramsey by compactness.

Definition 6.3. For $n \geqslant 2$, define the finite bipartite graphs $W_{n}$ and $M_{n}$ :

$$
\begin{aligned}
& W_{n}=\left\langle\left\{x_{i} \mid 1 \leqslant i \leqslant n\right\},\left\{y_{j} \mid 1 \leqslant j \leqslant n+1\right\},\left\{\left\langle x_{i}, y_{j}\right\rangle \mid j=i \text { or } j=i+1\right\}\right\rangle . \\
& M_{n}=\left\langle\left\{x_{i} \mid 1 \leqslant i \leqslant n+1\right\},\left\{y_{j} \mid 1 \leqslant j \leqslant n\right\},\left\{\left\langle x_{i}, y_{j}\right\rangle \mid j=i \text { or } j=i-1\right\}\right\rangle .
\end{aligned}
$$

Pictured are $W_{2}$ and $M_{3}$.
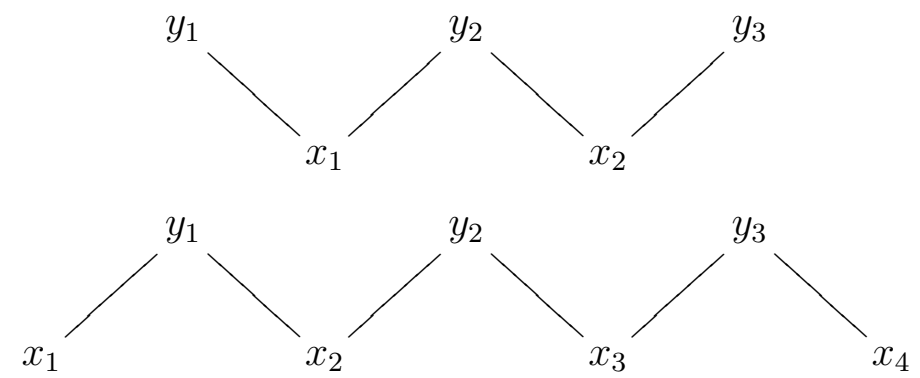

Lemma 6.4. Suppose $\mathcal{G}$ is an edge-Ramsey collection of finite bipartite graphs, closed under connected extensional subgraphs, containing $W_{n}$ but not $W_{n+1}$, and let $\mathbb{P}=\mathbb{P}(\mathcal{C} \ell(\mathcal{G}))$. Then $\mathcal{G}_{<}(\mathbb{P})$ contains $W_{n}$ and $M_{n+1}$ but not $W_{n+1}$ (and therefore not $M_{n+2}$ ).

Hence, $\mathcal{G}_{>}(\mathbb{P})$ contains $W_{n+1}$ but not $W_{n+2}$.

Proof. First, as $W_{n} \in \mathcal{G}$, by construction of $\mathbb{P}=\mathbb{P}(\mathcal{C} \ell(\mathcal{G}))$ there is some $p \in \mathbb{P}$ with $W_{n} \hookrightarrow$ $G_{[p]}$. That is, there are elements $p_{1}, \ldots, p_{n}$ in $[p]$, and there are elements $q_{1}, \ldots, q_{n+1} \in$ $\mathbb{P}$ such that $\left\langle\left\{p_{i}\right\},\left\{\operatorname{Pred}_{n}\left(q_{j}\right)\right\}, \in\right\rangle$ is a copy of $W_{n}$. But then, so is $\left\langle\left\{p_{i}\right\},\left\{q_{j}\right\},<_{\mathbb{P}}\right\rangle$, witnessing $W_{n} \in \mathcal{G}_{<}(\mathbb{P})$.

Now, consider $p_{i}, q_{j}$ as above. As the $q_{j}$ have different predecessors, they are incompatible. As $p_{i}$ and $p_{i+1}$ are incomparable but compatible, the $p_{i}$ all lie on the same level $n$. Choose any $r>_{\mathbb{P}} q_{1}$ and $r^{\prime}>_{\mathbb{P}} q_{n+1}$. Because $\mathbb{P}$ is $N 5$-omitting, $r$ and $r^{\prime}$ have the same predecessors on level $n$ as $q_{1}$ and $q_{n+1}$, respectively. Then

$$
\left\langle\left\{q_{1}, p_{1}, \ldots, p_{n}, q_{n+1}\right\},\left\{r, q_{2}, \ldots, q_{n}, r^{\prime}\right\},<_{\mathbb{P}}\right\rangle
$$

is a copy of $M_{n+1}$, witnessing $M_{n+1} \in \mathcal{G}_{<}(\mathbb{P})$.

Finally, by way of contradiction, suppose $\left\langle\left\{x_{p_{i}}\right\},\left\{y_{q_{j}}\right\},<_{\mathbb{P}}\right\rangle$ witnesses $W_{n+1} \in \mathcal{G}_{<}(\mathbb{P})$. Distinct $p_{i}$ must be incomparable because each has a successor not above the other. Because the $p_{i}$ are incomparable but $\equiv$-equivalent in $\mathbb{P}$, they are all in the same equivalence class $a \subseteq \mathbb{P}_{m}$. But then $\left\langle\left\{p_{i}\right\},\left\{\operatorname{Pred}_{m}\left(q_{j}\right)\right\}, \in\right\rangle$ realizes $W_{n+1}$ as a subgraph of $G_{a}$, contradicting our assumptions on $\mathcal{G}$. 
Proposition 6.5. There is an infinite sequence of distinct edge-Ramsey collections of finite bipartite graphs, $\left\langle\mathcal{G}_{n} \mid n \in \omega\right\rangle$, each closed under subgraphs and having the joint embedding property, and a corresponding sequence of pairwise non-biembeddable countably infinite chain-Ramsey partial orderings, $\left\langle\mathbb{P}_{n} \mid n \in \omega\right\rangle$.

Proof. Define

$$
\begin{gathered}
\mathbb{P}_{0}=2^{<\omega} ; \\
\mathcal{G}_{n}=\mathcal{G}_{>}\left(\mathbb{P}_{n}\right) \\
\mathbb{P}_{n+1}=\mathbb{P}\left(\mathcal{C} \ell\left(\mathcal{G}_{n}\right)\right) .
\end{gathered}
$$

An argument similar to that in the proof of Proposition 6.4 shows that $\mathcal{G}_{<}\left(2^{<\omega}\right)$ contains $M_{2}$ but not $W_{2}$, and therefore $\mathcal{G}_{0}$ contains $W_{2}$ but not $W_{3}$. It then follows from Proposition 6.4 that the $\mathcal{G}_{n}$ are distinguished from each other by the fact that $W_{n+2} \in \mathcal{G}_{n}$ but $W_{n+3} \notin \mathcal{G}_{n}$. Corollary 4.13 then implies that the $\mathbb{P}_{n}$ are pairwise non-biembeddable.

However, $\mathcal{G}_{n}$ is not characterized by omitting $W_{n+3}$, as there are other finite bipartite graphs it also omits. For instance, an argument similar to that in the proof of Proposition 6.4 shows that if a simple cycle of length greater than four is not in $\mathcal{G}$ (where $\mathcal{G}$ is closed under subgraphs), then it cannot be in $\mathcal{G}_{<}(\mathbb{P}(\mathcal{C} \ell(\mathcal{G}))$ ), and hence it cannot be in $\mathcal{G}_{>}(\mathbb{P}(\mathcal{C} \ell(\mathcal{G})))$. Therefore, every $\mathcal{G}_{n}$ omits every simple cycle of length greater than four.

\section{Questions}

We have given a complete characterization of the biembeddability classes of finite-level chain-Ramsey partial orderings.

(1.) Is there a finite-level partial ordering $\mathbb{P}$ such that $R^{2}(\mathbb{P})$ holds but $R^{3}(\mathbb{P})$ does not hold?

For a proto-Ramsey partial ordering $\mathbb{P}$, by Propositions 4.8 and Theorem $4.21, R^{2}(\mathbb{P})$ implies $R(\mathbb{P})$.

The only use of $R^{3}(\mathbb{P})$ in the proof that every finite-level chain-Ramsey partial ordering is biembeddable with a proto-Ramsey partial ordering is in Lemma 3.3 to the proof that a finite-level chain-Ramsey partial ordering with least element must be $N 5$-omitting. If $R^{2}(\mathbb{P})$ could be shown to suffice, it would then follow that $R^{2}(\mathbb{P})$ implies $R^{3}(\mathbb{P})$ (and, in fact, $R(\mathbb{P})$ ) for every finite-level partial ordering.

There are many examples of countably infinite partial orderings for which $R^{1}(\mathbb{P})$ holds but $R^{2}(\mathbb{P})$ does not. The essentially binary nature of a partial ordering might suggest that $R^{2}(\mathbb{P})$ should imply $R(\mathbb{P})$. On the other hand, $R^{2}(\omega)$ is proof-theoretically weaker than $R^{3}(\omega)$ (see $[12]$ ). This suggests that $R^{2}(\mathbb{P})$ might not suffice to imply $R^{3}(\mathbb{P})$.

(2.) Is there a partial ordering $\mathbb{P}$ with least element such that $R(\mathbb{P})$ holds but $\mathbb{P}$ is not $N 5$-omitting? 
Such an example could not be finite-level by Proposition 3.4. As any countably infinite chain-Ramsey partial ordering with least element must have an infinite chain, the assumption " $\mathbb{P}$ is finite-level" in Lemma 3.3 can be replaced by "P has a least element." Only the proof of Proposition 3.4, then, relies on the assumption that $\mathbb{P}$ is finite-level. The proof of Proposition 3.4 can be modified to show that if $\mathbb{P}$ is any countably infinite chain-Ramsey partial ordering with least element, possibly having infinite levels, then $\mathbb{P}$ omits the hexagon lattice.

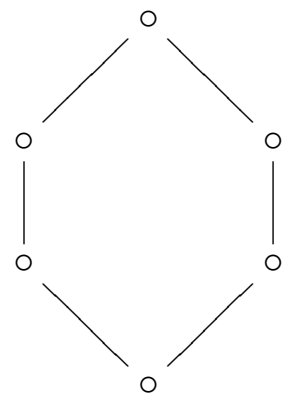

A negative answer to this question would show that all countably infinite $\omega$-linearizable chain-Ramsey partial orderings with least element (or, in fact, with any finite maximal antichain) are biembeddable with proto-Ramsey partial orderings, hence biembeddable with partial orderings $\mathbb{P}(\mathcal{G})$ for j.e.p. edge-Ramsey collections $\mathcal{G}$ of finite or countably infinite bipartite graphs.

(3.) What examples can be generated by modifications of the construction of Proposition 6.5?

For example, suppose we start with $\omega$ rather than $2^{<\omega}$. In the initial stages, we have

$$
\omega \hookrightarrow 2^{<\omega} \hookrightarrow \mathbb{P}\left(\mathcal{G}_{>}(\omega)\right) \hookrightarrow \mathbb{P}\left(\mathcal{G}_{>}\left(2^{<\omega}\right)\right)
$$

and none of these embeddings reverses, suggesting this might generate an entirely different sequence.

(4.) Find a complete characterization of the countably infinite chain-Ramsey partial orderings. That is, extend the characterization result Corollary 4.22 to all $\omega$-linearizable partial orderings.

As noted above, a negative answer to question (2) above would lead to a characterization of countably infinite chain-Ramsey partial orderings that have a least (or a greatest) element.

The (possibly) larger class of connected countably infinite chain-Ramsey partial orderings might be a next step. For a chain-Ramsey $\mathbb{P}$ not to be biembeddable with a partial ordering with least element, it is necessary (and sufficient) that $\mathbb{P}$ has no base (in the sense of Definition 3.7); if $\mathbb{P}$ is $\omega$-linearizable, it is necessary that $\mathbb{P}$ has no finite antichain.

There are many countably infinite chain-Ramsey partial orderings with infinitely many connected components. For example, if $\mathbb{P}$ is any countably infinite chain-Ramsey partial 
ordering with least element, then the disjoint union of the finite suborders of $\mathbb{P}$ is itself chain-Ramsey; if $\mathbb{P}$ is proto-Ramsey and has incompatible elements, any number of connected components isomorphic to $\mathbb{P}$ may be adjoined to get another chain-Ramsey partial ordering, although this one is biembeddable with $\mathbb{P}$. As another example, the disjoint union of the $\mathbb{P}_{n}$ constructed in Proposition 6.5 is a chain-Ramsey partial ordering; this has infinite connected components, but is not biembeddable with any connected partial ordering. As a third example, the fact that for any natural numbers $n$ and $k$ and any finite partial ordering $P$ there is a finite partial ordering $Q$ with $Q \rightarrow(P)_{k}^{n}$ ([7],[8]) shows that the disjoint union of all finite partial orderings is chain-Ramsey; because this collection contains $N 5$, it does not arise as the collection of finite suborders of any proto-Ramsey partial ordering. The disparity of these examples suggests that the general question might be more difficult than the question of connected partial orderings.

(5.) For other collections $\mathcal{A}$ of finite partial orderings, consider the question of which countably infinite partial orderings $\mathbb{P}$ satisfy $(\forall A \in \mathcal{A})\left[\mathbb{P} \rightarrow(\mathbb{P})^{A}\right]$.

Proposition 4.23 showed that if $\mathcal{A}$ is the collection of all finite partial orderings, then up to biembeddability, there are only five countably infinite $\mathbb{P}$ with this property. Section 1 suggested two reasons that many more countably infinite partial orderings are chainRamsey: first, chains have no non-rigid suborderings; second, chains are among a small class of $A$ for which, given any natural number $k$ and any finite partial ordering $P$, there is a finite partial ordering $Q$ with $Q \rightarrow(P)_{k}^{A}[7],[8]$. The first property appears to be the more salient. The second property also applies to chain sums of antichains [7],[8] (that is, finite suborderings of $M$ ult $(\omega))$; however, the only proto-Ramsey partial orderings that satisfy $\mathbb{P} \rightarrow(\mathbb{P})^{A}$ even for a two-element antichain $A$ are $\omega$ and $\operatorname{Mult}(\omega)$. There is, however, at least one example of a countably infinite partial ordering $\mathbb{P}$ with $\mathbb{P} \rightarrow(\mathbb{P})^{A}$ if $A$ is a chain-sum of antichains, but not if $A$ is an arbitrary finite partial ordering; namely, the disjoint union of finite chains of arbitrary length.

(6.) Following the lead of Nešetřil [9],[10], and noting that the proof of Proposition 4.23 depends partly on the interplay between the ordering $\leqslant \mathbb{P}$ and the natural ordering $\leqslant$ of the elements of $\mathbb{P}$ (assuming $\mathbb{P} \subseteq \omega$ ), consider countably infinite partial orderings $\mathbb{P}$ augmented with a linearization $\leqslant$ of $\leqslant \mathbb{P}$ in order type $\omega$.

If we ask, in the context of these augmented structures, which countably infinite $\mathbb{P}$ satisfy $\mathbb{P} \rightarrow(\mathbb{P})^{A}$ for all finite $A$, we get no new proto-Ramsey partial orderings beyond $\omega$ and $\operatorname{Mult}(\omega)$, but again we get at least one new countably infinite partial ordering beyond the five of Proposition 4.23, namely the disjoint union of finite chains of arbitrary length (with a suitable linearization).

(7.) Find a complete characterization, phrased in terms of omitted subgraphs, of the edge-Ramsey, j.e.p. collections of finite bipartite graphs of the form $\mathcal{C} \ell(\mathcal{G})$ 
This is a long-standing open problem. In a sense, the characterization results Theorem 4.21 and Corollary 4.22 of this paper are incomplete without an answer to this question.

\section{References}

[1] J. Chubb, J. Hirst and T. McNicholl. Reverse mathematics, computability, and partitions of trees. J. Symb. Logic 74(1):201-215, 2009.

[2] J. Corduan. Coloring Posets and Reverse Mathematics. Ph.D. thesis, Dartmouth College, 2011.

[3] J. Corduan, M. Groszek and J. Mileti. Reverse Mathematics and Ramsey's Property for Trees. J. Symb. Logic 75(3):945-954, 2010.

[4] P. Erdős, A. Hajnal and L. Pósa. Strong embeddings of graphs into colored graphs. In Infinite and Finite Sets, ed. A. Hajnal, R. Rado and V.T. Sós, Colloq. Math. Soc. János Bolyai 10:585-595, 1975.

[5] W.L. Fouché. Chain partitions of ordered sets. Order 13(3):255-266, 1996.

[6] K.R. Milliken. A Ramsey theorem for trees. J. Combin. Theory Ser. A 26(3):215-237, 1979.

[7] J. Nešetřil, and V. Rödl. Partitions of finite relational and set systems. J. Combin. Theory Ser. A 22(3):289-312, 1977.

[8] J. Nešetřil, and V. Rödl. Combinatorial partitions of finite posets and latticesRamsey lattices. Algebra Universalis 19(1):106-119, 1984.

[9] J. Nešetřil. Ramsey Theory. In Handbook of Combinatorics, ed. R.L. Graham, M. Grötschel, and L. Lovász, North-Holland, pages 1331-1403, 1995.

[10] J. Nešetřil. Ramsey Classes and Homogeneous Structures. Combinatorics, Probability, and Computing 14:171-189, 2005.

[11] F.P. Ramsey. On a problem of formal logic. Proc. London Math. Soc. Ser. 2 30:264286, 1930.

[12] S. Simpson. Subsystems of Second Order Arithmetic. Perspectives in Mathematical Logic, Springer-Verlag, Berlin, 1999. 